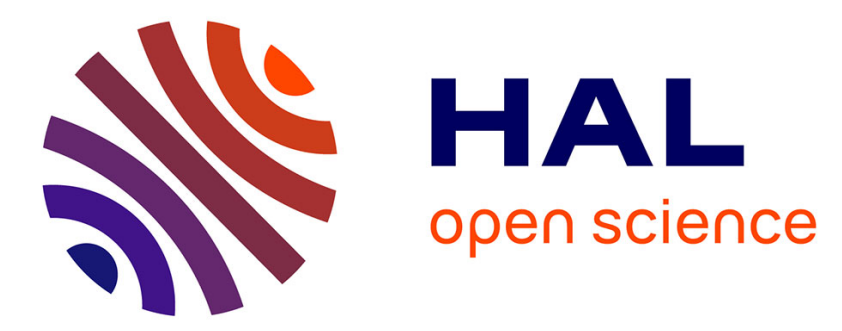

\title{
Philosophes du langage et autonymie: Une déjà longue histoire
}

Philippe de Brabanter

\section{To cite this version:}

Philippe de Brabanter. Philosophes du langage et autonymie: Une déjà longue histoire. Histoire Epistémologie Langage, 2005, 27 (1), pp.1-30. ijn_00000600

\section{HAL Id: ijn_00000600 \\ https://hal.science/ijn_00000600}

Submitted on 4 Apr 2005

HAL is a multi-disciplinary open access archive for the deposit and dissemination of scientific research documents, whether they are published or not. The documents may come from teaching and research institutions in France or abroad, or from public or private research centers.
L'archive ouverte pluridisciplinaire HAL, est destinée au dépôt et à la diffusion de documents scientifiques de niveau recherche, publiés ou non, émanant des établissements d'enseignement et de recherche français ou étrangers, des laboratoires publics ou privés. 


\title{
PHILOSOPHES DU LANGAGE ET AUTONYMIE : UNE DEJA LONGUE HISTOIRE ${ }^{1}$
}

\author{
Philippe De Brabanter \\ Institut Jean Nicod, CNRS-EHESS-ENS
}

Résumé: Nous tentons de montrer la richesse de la réflexion sur l'autonymie en philosophie du langage et de mettre cette réflexion en parallèle avec l'évolution de la discipline au cours des cent-vingt dernières années. Les philosophes-logiciens de la fin du 19è et première moitié du 20è siècle (citons Frege, Tarski et Carnap) aspiraient à construire, à l'usage de la science, des langages formels dépourvus des défauts des langues ordinaires. Mais le projet logiciste s'est révélé irréalisable et les préoccupations se sont déplacées des systèmes formels vers les langues naturelles et le langage. Dans le même temps, s'est substituée à un discours plutôt prescriptif une entreprise de description et d'analyse proprement linguistique, centrée principalement sur les phénomènes qui mettent en jeu la signification. Les théories avancées pour caractériser l'autonymie reflètent cette transformation de la discipline : on est passé de propositions (l'autonyme comme nom ou comme description définie) qui convenaient aux langages formels à des théories qui épousent mieux la diversité des manifestations réelles de l'autonymie. Ces théories permettent entre autres de rendre compte, avec une réussite variable, des cas nombreux où un fragment de discours semble être à la fois en emploi autonyme et en emploi ordinaire (cf. Ton "amoureux» est arrivé). Ces énoncés hybrides sont importants parce qu'ils touchent à des questions centrales en philosophie du langage, notamment le problème de la frontière entre sémantique et pragmatique.

Abstract: This paper reviews the wealth of theories put forward by philosophers of language over the last 120 years to account for autonymy (= the mention of linguistic expressions), and relates these theories to the major developments that the discipline has gone through. The philosophers-cum-logicians from the turn of the 19th and 20th century (notably, Frege, Tarski and Carnap) hoped to build reliable formal languages for science that would not exhibit the flaws of natural languages. But their logicist project proved unfeasible and interest shifted from formal systems to language and ordinary speech. The largely prescriptive judgments of philosophers of language concurrently gave way to description and analysis of linguistic phenomena, with special focus on meaning. Theories of autonymy bear witness to these transformations: from proposals tailored to the characteristics of formal languages (autonyms as names or definite descriptions), they have evolved into accounts that allow for the diversity of autonymy-

\footnotetext{
${ }^{1}$ Nous remercions Irène Rosier-Catach, Claude Panaccio, Sarah Pialeprat, ainsi que les autres participants à ce numéro de H.E.L. pour leurs commentaires sur une version antérieure du présent texte. Nous tenons également à reconnaître notre dette à l'égard du travail de repérage et de mise en perspective réalisé par Josette Rey-Debove (1978) et (1997b). Précisons toutefois que l'exposé de Rey-Debove sur les conceptions de l'autonymie chez les logiciens s'arrête aux années septante alors que nous couvrons le sujet jusqu'en 2004.
} 
related phenomena. These theories provide explanations - some better than others - for the numerous instances of "simultaneous use and mention" of an expression (cf. Your "sweetheart" has arrived). Such hybrid utterances are important because they affect our ways of looking at some central issues in the philosophy of language, notably that of the semantics/pragmatics interface.

Mots-clés : autonyme, citation, mention, guillemets, iconicité, sémantique, pragmatique

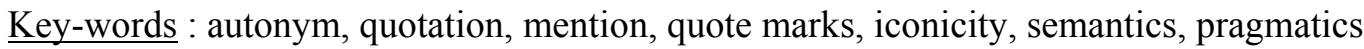

\section{Introduction ${ }^{2}$}

Il peut être utile, lorsqu'on souhaite parler de philosophie du langage à un public composé essentiellement de linguistes et d'historiens de la linguistique, d'aider son lecteur à se faire une idée du mode de fonctionnement de cette discipline. La philosophie du langage a, de nos jours, des préoccupations proches de la linguistique théorique, en particulier de la sémantique et pragmatique formelles. Nous pensons ici surtout à cette manière de pratiquer la linguistique, fortement empreinte de logique et de formalisation, qui est typique des Anglo-saxons et de ceux qui s'inspirent de leurs modèles. Les philosophes du langage recourent en général à la méthode dite de l'« analyse », qui doit être comprise, au sens de la philosophie analytique, comme mettant l'accent sur la structure logique des choses (faits, actions, concepts) et qui est censée sous-tendre des hypothèses construites de manière à être réfutables, comme dans les sciences naturelles.

\footnotetext{
${ }^{2}$ Un mot sur nos conventions typographiques. Pour les autonymes employés aux fins de citation métalinguistique, nous utilisons l'italique :

Beau est un adjectif.

Pour citer pareil autonyme, nous le plaçons entre guillemets français :

Dans la phrase précédente, «Beau » est un autonyme.

Pour le discours direct, la citation mixte, le scare quoting (notions expliquées plus loin) et l'introduction de termes techniques, nous utilisons les guillemets français :

Saf a acheté une « mob ».

Pour citer pareille citation, nous la mettons en italique, guillemets inclus :

Dans cette phrase, " $m o b$ » ne désigne pas un mot mais un véhicule.

Il faut toutefois faire attention au fait que, dans les citations d'auteurs, nous n'avons pas modifié la typographie originale. Ainsi, si un auteur cité emploie les guillemets simples (anglais) pour indiquer l'autonymie :

'Boston' rhymes with 'Thurston',

nous avons respecté cette convention. Ceci implique que, lorsque nous voulons référer à l'un des autonymes qui apparaissent dans cette phrase, nous devons le mettre en italique, y compris les guillemets :

Cette phrase a pour sujet 'Boston', qui est autonyme.

Nous sommes conscients que tout ceci est compliqué et laborieux. Mais il n'y a pas de solutions simples dès lors qu'il s'agit de marquer des niveaux métalinguistiques.
} 
La philosophie du langage, dont la plupart des spécialistes s'accordent à dire qu'elle est née avec les réflexions de Frege durant le dernier quart du 19è siècle, a connu en plus de cent ans plusieurs évolutions. Au départ, de leur étude des langues naturelles, les philosophes en concluaient généralement à leur inadéquation foncière pour le but qu'ils poursuivaient, à savoir la mise au point de notations leur garantissant des "langages bien en ordre », c'est-à-dire des outils expressifs non ambigus et non contradictoires, utiles à la construction d'une «langue de la science» et permettant de mettre à nu et d'éviter toutes sortes de "faux problèmes" métaphysiques. Nombreux, chez ceux qui souscrivaient à ce programme, sont les commentaires dénonçant les défauts des langues ordinaires (notamment, Frege 1892 : 28-29 ; Russell 1919 : 172, Carnap 1937 : 2; Tarski 1944 : 347).

On voit bien que la réflexion linguistique des philosophes de l'époque est de nature davantage prescriptive que descriptive. Il en sera ainsi jusque dans les années cinquante, époque qui voit naître une nouvelle pratique, la " philosophie du langage ordinaire », qui, avec des personnalités telles qu'Austin, Strawson et Grice, se penche résolument sur l'usage ordinaire des langues. Ce tournant oriente les philosophes vers la description et l'explication du fonctionnement des langues naturelles. Enfin, depuis une bonne trentaine d'années, en raison de la floraison de théories issues des travaux de Chomsky, la philosophie du langage s'est encore rapprochée de la linguistique, et n'est souvent plus clairement distincte de la sémantique formelle et de la pragmatique linguistique, deux disciplines que beaucoup de praticiens s'efforcent d'articuler aux autres branches de la linguistique, notamment à la syntaxe.

Nous verrons que la réflexion sur l'autonymie reflète les infléchissements qu'a connus la philosophie du langage. À l'origine, l'intérêt des pères fondateurs de la discipline pour l'autonymie (qui ne s'appelle pas encore comme ça) se porte sur le moyen d'empêcher de confondre l'emploi ordinaire d'une expression langagière (souvent aux fins de désigner une entité «mondaine ») et l'emploi, moins ordinaire, d'une expression dans le but de faire référence à cette expression même (ou à une expression qui lui ressemble fortement). En gros, il s'agissait d'indiquer clairement si l'on parlait de la chose ou du mot.

Cette distinction s'intègre dans une réflexion plus large sur le couple « langage-objet/métalangage ». Le tournant du 19è au 20è siècle voit naître plusieurs tentatives pour donner un fondement logique à l'une ou l'autre branche des mathématiques. Des gens comme Gottlob Frege et David Hilbert entreprennent donc de construire un langage (un système formel) dans lequel exprimer les axiomes de l'arithmétique. A cette fin, ils en viennent progressivement à se servir d'un autre langage (souvent une langue naturelle augmentée de symboles logiques), qui leur permet notamment d'énoncer les symboles du système axiomatique et les règles de bonne formation des formules composées de ces symboles. C'est ainsi qu'apparaît, d'abord de manière informelle, la distinction entre langage-objet (par exemple, une axiomatisation de 
l'arithmétique) et métalangage (par exemple, l'allemand de Frege et Hilbert augmenté de quelques symboles logiques).

Au départ, on croyait, et l'on espérait, qu'il serait possible d'exprimer dans le langage servant à formaliser l'arithmétique (pour prendre un exemple) certaines caractéristiques essentielles à une axiomatisation réussie, telles que la complétude et la consistance du système. On pensait donc pouvoir se contenter de démonstrations entièrement formelles, autrement dit, purement «syntaxiques ». Mais, suite à la démonstration de l'incomplétude de l'arithmétique par le mathématicien Kurt Gödel en 1931, on dut admettre que, pour établir la complétude et la consistance d'un système formel (d'une « puissance » égale ou supérieure à l'arithmétique), il était nécessaire de recourir à un langage d'ordre supérieur, un métalangage qui constitue la "sémantique " $\mathrm{du}$ système en question. Ce résultat rendait d'autant plus indispensable une séparation nette entre les énoncés appartenant au langage-objet et ceux qui ressortissaient au métalangage. ${ }^{3}$ C'est à ce prix seulement que l'on pouvait s'assurer que le système formel ne succomberait pas aux mêmes "paradoxes de l'autoréférence » - par exemple, l'antinomie du menteur (Ce que je dis est faux) - qui entachaient l'usage des langues naturelles.

\section{Carnap et le baptême de l'autonyme}

La tradition veut que Gottlob Frege soit le premier à avoir insisté sur l'usage des guillemets (« Anführungszeichen ») pour distinguer l'emploi autonymique de l'emploi ordinaire (cf. Imbert 1971 : 19; Bynum $1972: 33,84$ note 4). ${ }^{4}$ Mais ce n'est pas lui qui créa le mot autonyme. Ce privilège revient à Rudolf Carnap, l'un des plus célèbres représentants du positivisme logique. En 1934, il publie sa Logische Syntax der Sprache ${ }^{5}$ livre dans lequel apparaît pour la première fois l'adjectif/adverbe autonym (la traduction anglaise recourt au couple autonymous/autonymously). La définition non formelle la plus détaillée que Carnap offre d'une expression en emploi autonyme est : "Lorsqu'un symbole [du métalangage] s'utilise ainsi comme nom de lui-même (ou, plus exactement, comme nom de sa propre forme), nous le qualifions d'autonyme » (1934:16, 1937 : 17).

Quelques précisions s'imposent: tout d'abord, Carnap n'utilise pas de substantif Autonym: il n'y a d'autonymes que des emplois particuliers d'une

\footnotetext{
${ }^{3}$ Ces étiquettes sont «sensibles au contexte », puisqu'un métalangage peut lui-même devenir l'objet d'une étude ou d'une construction qui sera réalisée à l'aide d'un métalangage de second ordre, ou métamétalangage.

${ }^{4}$ Les deux passages clés chez Frege sont à la page 28 de Über Sinn und Bedeutung (1892) [p. 104 de l'édition française des Ecrits logiques et philosophiques] et à la page 4 du premier volume des Grundgesetze der Arithmetik (1893).

5 Traduction anglaise : The Logical Syntax of Language, traduit par Amethe Smeaton (Comtesse Von Zeppelin), Londres, Routledge \& Kegan Paul, 1937. Nous n'en connaissons pas de traduction française.
} 
expression. Ceci suggère qu'il n'existe pas pour Carnap d'autonymes dans le lexique - ils apparaissent uniquement dans le contexte d'une phrase. Ensuite, il s'agit de comprendre pourquoi Carnap rectifie sa formulation initiale l'autonyme comme nom de lui-même - et écrit que l'expression autonyme désigne plutôt sa propre forme. C'est ainsi que nous avons traduit le mot Gestalt [la traductrice anglaise a choisi symbol-design]. Les commentateurs de Carnap semblent s'accorder sur le fait que Gestalt dénote ici la même idée que le terme de type chez Peirce. ${ }^{6}$ L'expression en emploi autonyme est donc une occurrence qui désigne son type. Cette différence entre l'expression «mentionnante » et l'expression "mentionnée », couplée au fait que l'expression mentionnante est qualifiée de «nom », semble indiquer que l'autonyme et sa désignation ne sont pas une seule et même chose. Cette conclusion est toutefois contrariée par l'utilisation fréquente du pronom réfléchi sich selbst (lui-même) et par le fait que Carnap indique $(1934: 107 ; 1937$ : 154-55) que l'autonyme peut désigner non seulement un type, mais aussi une occurrence déterminée dans le temps et l'espace (un «token »). Mais, même dans ce second cas, il n'est pas clair que l'autonyme doive se comprendre comme nom de lui-même au sens strict du terme, puisque la référence peut être à un autre token que celui qui figure dans l'énoncé de la phrase.

Ces quelques hésitations de Carnap mettent en lumière au moins deux questions qui tracasseront les philosophes au 20è siècle: (i) A quoi réfère un autonyme (à lui-même, au type qu'il instancie, à l'un ou à l'autre, à d'autres choses encore)? (ii) L'autonyme n'est-il qu'un emploi particulier d'une expression ou est-il une expression différente de celle qu'il désigne ? J'aborderai brièvement la première ici-même ; quant à la seconde, elle est centrale à toutes les grandes théories de l'autonymie avancées au cours des cent dernières années ; nous aurons donc pleinement l'occasion d'y revenir. ${ }^{7}$

Toute tentative de réponse à la question (i) est délicate parce qu'elle dépend en partie de la théorie de l'autonymie que l'on défend - la cohérence interne impose des contraintes - et de la conception de la référence que l'on adopte. Nous ne pourrons ici faire mieux qu'énoncer les thèses en présence. Premièrement, il y a ceux qui écrivent qu'un autonyme " réfère à lui-même ". Mais nombre d'entre eux ne voient vraisemblablement ici qu'un raccourci d'expression (p.ex. Garver 1965: 235; Seymour 1996: 309). Devraient cependant appartenir à ce groupe les tenants de ce que nous appellerons par la suite la "Théorie de l'identité » (Christensen, Searle, Recanati 1979, Washington), mais, curieusement, soit ils nient que l'autonyme réfère, soit ils récusent l'idée que l'autonyme se désigne en tant que «ce token-ci dans le

\footnotetext{
${ }^{6}$ Dans son Introduction to Semantics, rédigé par lui-même en anglais, Carnap opère une distinction entre "sign-event» et "sign-design» qui est l'analogue de la distinction entre « token » et « type » chez Peirce. (Voir Carnap $1948: 5-8$ )

${ }^{7}$ On notera avec intérêt qu'elle se posait déjà à l'époque médiévale, sous la forme de l'opposition entre univocatio (emploi d'une forme avec plusieurs sens) et equivocatio (emploi de plusieurs formes pour exprimer plusieurs sens). Voir Rosier-Catach (2004).
} 
présent énoncé ». Deuxièmement, on distingue un groupe, majoritaire, qui estime que l'autonyme renvoie (quasi) toujours à un type ou à une classe de tokens. Se côtoient ici des "nominalistes" (Tarski, Carnap), un théoricien de l'identité (Washington 1998 : 550), ainsi que Donald Davidson et la majorité des auteurs qui ont adopté/adapté sa théorie « démonstrative » (Bennett, Reimer, Cappelen \& Lepore, Recanati 2001). Enfin, il y a ceux qui pensent que l'autonyme peut référer à une variété d'objets, selon le contexte. Citons Carnap (!), Saka (1998) et De Brabanter (à paraître). Les arguments sont trop complexes pour être détaillés ici, mais nous ne pouvons résister à proposer à notre lecteur quelques exemples susceptibles d'exercer sa sagacité. Il peut être utile de prêter une attention particulière aux prédicats métalinguistiques et de recourir à l'épreuve de la traduction (les autonymes pertinents sont en caractères gras) :

(1) a. Pouvoir est ici écrit à l'encre noire, en italique, et avec une capitale.

b. Pouvoir est à la fois un verbe et un nom.

c. Pouvoir se traduit en anglais par can.

d. ?? Pouvoir est à la fois un verbe et un nom et se traduit en anglais par can.

e. Pouvoir, un infinitif, s'oppose à peux, peut, pouvons, etc., qui sont des formes conjuguées.

f. Pouvoir prend les formes peux, peut, pouvons, etc., selon la personne et le nombre.

g. ?? Pouvoir, un infinitif, s'oppose à peux, peut, pouvons, etc., qui sont des formes conjuguées, et prend les formes peux, peut, pouvons, etc., selon la personne et le nombre.

h. A son oreille, la voix lui crie:

- Fais gaffe, ralentis, t'es dingue ou...

L'inconnu n'a pas le temps de terminer sa phrase car Albert a donné un brusque coup de volant vers la gauche. (http://www.lapoesie.com/atelier2b.html)

i. Son anglais se résumait à ceci: “\#\$\%@\#\&!@”. (notre traduction d'une phrase trouvée sur www.marknair.com/2002/11/)

Nous donnons quelques indications (toutes discutables) : l'énoncé (a) oblige (à cause de $i c i$ ) à se demander si un signe-token peut renvoyer à lui-même en tant que token (problème de la sui-référentialité). Le "Pouvoir» de (b) semble désigner une forme abstraite (plutôt qu'un token dans un énoncé ou que le lexème pouvoir: ni l'un ni l'autre ne pourrait être à la fois nom et verbe). Le "Pouvoir » de (c), en revanche, semble désigner le lexème verbal, puisque le nom pouvoir ne se traduit pas par can. L'acceptabilité douteuse de la conjonction d'énoncés en (d) semble confirmer que les sujets de (b) et (c) dénotent des référents distincts. Il est toutefois possible que ces référents soient des types (de nature différente). De manière similaire, l'acceptabilité douteuse de la 
conjonction d'énoncés en $(\mathrm{g})$ suggère que les «Pouvoir » de $(\mathrm{e})$ et $(\mathrm{f})$ désignent des entités différentes: une forme verbale en (e), un lexème en (f). Encore une fois, il se peut que ces deux référents soient des types (mais des types distincts). L'autonyme en (h) désigne des paroles prononcées précédemment (par «la voix »). On est tenté de dire que de telles paroles ne peuvent être qu'un token, puisqu'elles sont situées dans le temps et l'espace. De plus, l'incomplétude du rapport de discours pose un problème supplémentaire pour le partisan du « toutau-type ». Si l'on comprend bien qu'une phrase-token soit une occurrence, parmi d'autres, d'une phrase-type, c'est moins clair dans le cas de l'énoncé interrompu : qu'est-ce en effet que le type d'un énoncé interrompu ? Enfin, que dénote le bizarroïde autonyme de (i) ? Token, type, autre chose ? Les intuitions sont moins claires à mesure qu' on s'éloigne des exemples de base.

\subsection{Postérité de la terminologie de Carnap}

Le terme d' "autonyme » n'a pas connu un grand succès, en tout cas dans le monde de la linguistique et de la philosophie anglo-saxonnes. Peu nombreux sont les auteurs à s'être réapproprié ce terme (bien moins par exemple que ceux qui font mention du terme scolastique de suppositio materialis). On notera, rare exception, le cas d'Alonzo Church, dont l'usage du terme d'autonyme est fidèle à Carnap (cf. 1956 : 61), excepté qu'il emploie (et crée ?) les substantifs autonym et autonymy (ibid. 62-63). Pour le reste, le terme est peu usité et, souvent même, n'apparaît pas dans les dictionnaires et encyclopédies de linguistique et de philosophie. ${ }^{8}$ La situation est différente en Allemagne, pays de naissance de Carnap, et plus encore en France, où autonyme et ses dérivés ont été largement popularisés par les travaux de Josette Rey-Debove (1978) ${ }^{9}$ et sont d'usage courant en linguistique (on notera toutefois que Rey-Debove modifie profondément la définition de Carnap). On appréciera enfin le fait que Carnap lui-même n'a que très peu recouru au terme dans ses écrits postérieurs à 1934. Il n'y a, à ma connaissance, que dans Meaning and Necessity que le terme apparaisse, et encore une seule fois (1947: 4).$^{10}$ Il semble bien que Carnap ait fini par renoncer entièrement à son néologisme : on en veut pour preuve ces passages où il décrit l'emploi autonyme, ou encore ses propres conventions pour la citation d'expressions, sans pourtant jamais faire usage du terme d' " autonyme » (notamment $1948: 237,1950: 56-57$ ).

\footnotetext{
${ }^{8}$ De manière assez remarquable, le terme ne figure meme pas dans l'index des meilleures encyclopédies de philosophie en anglais, Edwards (1967) et Craig (1998), ou dans celui de la Concise Encyclopedia of Philosophy of Language de Lamarque.

${ }^{9}$ En Allemagne, les gros ouvrages de Ritter (1971-2004) et Mittelstraß (1995-96) consacrent chacun un article à l'adjectif/adverbe autonym. En France, le terme bénéficie d'une entrée dans l'Encyclopédie universelle de la philosophie; l'article est de la plume de Rey-Debove.

${ }^{10}$ Edition française, avec une introduction : Signification et Nécessité, traduit par F. Rivenc et Ph. de Rouilhan, Editions Gallimard, 1997.
} 


\section{Emploi et mention chez Quine}

Carnap n'était ni le seul ni le premier philosophe à proposer une Théorie de l'autonyme comme nom. ${ }^{11}$ Quelques années auparavant, en 1931, le grand logicien polonais Alfred Tarski avait donné un tableau très semblable de l'autonyme (que son traducteur anglais nommait quotation-mark name). Et dès le milieu du 19è siècle, J.S. Mill avait déjà énoncé cette même idée de l'autonyme comme nom dans son System of Logic (1862: I, 24), sans toutefois la développer.

Le partisan le plus connu de la Théorie nominale est W.V.O. Quine, et ses positions ont eu un écho bien plus durable que celles des autres auteurs évoqués ici. Ceci est sans doute dû au fait qu'il a introduit, en 1940, dans son Mathematical Logic, ${ }^{12}$ un couple terminologique qui va faire florès, tant en linguistique qu'en philosophie : use et mention. ${ }^{13}$ Ces termes sont encore d'usage courant de nos jours, mais il faut savoir qu'ils s'entendent dans des sens différents (et parfois incompatibles) d'un auteur à l'autre. On peut par ailleurs penser que le succès du terme mention n'est pas étranger à la postérité mitigée d'autonyme et de ses dérivés.

Comment Quine définit-il son couple terminologique? Prenons les trois premiers exemples pertinents du livre de 1940 :

(2) Boston is populous

(3) 'Boston' is disyllabic

(4) Boston is disyllabic

En (2), nous dit Quine, il y a emploi de Boston à la fin de mentionner une ville. En (3), c'est le nom 'Boston' (avec les guillemets !) qui est employé à la fin de mentionner le nom Boston (sans les guillemets). On le voit, contrairement à ce que l'on peut lire dans certains exposés sommaires, il n'y a aucune incompatibilité entre emploi et mention: la mention d'une chose nécessite toujours l'emploi d'une expression.

On notera que, peu soucieux des réalités de l'usage, Quine stipulait que les exemples comme (4) sont faux, puisqu'ils attribuent une propriété linguistique à un objet (ici, une ville) qui n'en a pas. Donc, il est nécessaire, lorsqu'on veut mentionner une entité linguistique, de recourir aux guillemets (ou à une autre marque de l'autonymie). En d'autres termes, il est nécessaire de produire une citation (quotation) de l'expression mentionnée. La citation, au sens de Quine, est donc un nom qui mentionne une entité linguistique.

\footnotetext{
${ }^{11}$ Chez tous les auteurs anglophones dont il est question ici, ainsi que dans les traductions anglaises des non-anglophones, on a le mot name, qui désigne un nom propre (par opposition à noun, qui désigne un nom commun).

12 À ma connaissance, il n'en existe pas de traduction française.

${ }^{13}$ Termes que nous traduirons par emploi et mention (même si d'autres préfèrent usage pour le premier).
} 
La conception nominale de l'autonymie allait faire office de doxa en philosophie du langage pendant près de trente ans. Elle est d'ailleurs encore tout à fait d'actualité en logique, où l'adéquation empirique de la théorie n'est pas un enjeu. Le travail de sape commencera dans les années soixante (Garver 1965, Christensen 1967), avec, en apothéose, la critique brève mais incisive que Searle donnera dans Speech Acts (1969: 73-76). ${ }^{14}$

\subsection{L'autonyme comme nom : critiques}

Nous reprenons rapidement les principales objections de Searle. Son premier argument est que l'on n'a pas besoin d'user d'un nom lorsque le porteur du nom " est lui-même un segment de discours et est donc facile à produire et ne requiert pas un mécanisme linguistique séparé pour y référer » $(1969: 75$; le même argument apparaît chez Sørensen 1961 : 189; Christensen 1967 : 360 ; Recanati 1979 : 68). Mais le raisonnement de Searle n'est pas tout à fait probant. Que veut dire Searle lorsqu'il affirme que "l'objet dont nous souhaitons parler» est présent dans l'énoncé ? L'objet qui est présent, c'est un token d'une expression. Rien ne prouve toutefois que ce soit de ce token que l'on souhaite parler. (Je reviendrai sur cette question dans la section 5 , consacrée à la «Théorie de l'identité ».)

La seconde critique de Searle aux tenants de la Théorie du nom est qu'ils ne voient pas que les phrases (2) et (3) commencent par le même mot. Searle lie cet aveuglement des « philosophes et logiciens » à leur conception selon laquelle les noms propres de mots se forment par adjonction de guillemets. Suit un passage destiné à illustrer l'absurdité du procédé (par souci de clarté, nous avons remplacé le nom Socrates de l'exemple de Searle par Boston) :

Dans cette conception, le premier mot de [(3)] n'est pas, comme vous pourriez le penser, «Boston», mais « «Boston » ». Et, ce qui est assez difficile à saisir, le mot que je viens d'écrire n'est pas « «Boston»», mais « « «Boston»»», lequel mot tout à fait nouveau est à son tour nom propre d'un nom propre de nom propre, à savoir « « « «Boston »»»». Et ainsi de suite dans la hiérarchie des noms de noms... (1969: 74).

Si l'on peut, avec Searle, estimer que pareille prolifération de noms propres n'est pas souhaitable, l'argument ne suffit pas à ébranler le nominaliste placide. De plus, qu'on le veuille ou non, la réitération des guillemets est indispensable à une présentation claire des données du métalangage naturel. Nous n'avons pas, dans le présent travail, procédé autrement que le nominaliste de l'exemple de Searle.

La troisième objection, qui s'appuie sur le fonctionnement sémantique des autonymes et des noms, est plus décisive. Il n'y a d'ordinaire aucune ressemblance entre un nom et ce qu'il nomme. Le nom Marvin ne ressemble pas

${ }^{14}$ Traduction française : Les Actes de langage, trad. H. Pauchard, Éditions Hermann, 1972. 
à l'individu Marvin, et tous les Marvin entre eux ne se ressemblent pas non plus. Autrement dit, à l'exception de certains noms de lieux et de certains surnoms (Gras-double, Ficelle, etc.), le lien entre un nom et son porteur est purement conventionnel. Dans l'autonymie, en revanche, la ressemblance entre expression mentionnée et expression mentionnante est flagrante : le lien est iconique. Pour qu'un autonyme soit vraiment un nom, suggère Searle avec malice, il faudrait que son rapport à son référent soit arbitraire. Ce serait le cas si John était le nom de Socrate (le nom, pas l'individu !). On pourrait ainsi dire, correctement, John a sept lettres. Manifestement, ce n'est pas ainsi que fonctionne l'autonymie. ${ }^{15}$ Paul Saka a tiré une intéressante conséquence de la dimension iconique de l'autonyme, conséquence valable à tout le moins pour une majorité de cas : « Nous pouvons passer, de manière productive, de la connaissance d'une expression quelconque à celle de sa citation [et] nous pouvons passer de la connaissance d'une citation quelconque à celle de l'expression elle-même » $(1998: 115) .^{16}$

Searle n'avait pas cru utile de présenter davantage d'objections dans Speech Acts. Mais, en 1983, dans Intentionality, ${ }^{17}$ il avançait un quatrième contreargument très intéressant. Searle observe que les citations apparaissent souvent dans des positions qui ne sont pas ouvertes aux noms (aux syntagmes nominaux) :

[...] notons la différence entre "Gerald says : 'I will consider running for the Presidency" " et "Gerald said he would 'consider running for the Presidency" . (1983: 185)

La citation, dans la seconde phrase, ne peut être un nom propre : si nous insérons, par exemple, Henry après would, nous obtenons Gerald said he would Henry, énoncé agrammatical et ininterprétable. ${ }^{18}$ En fait, ce qui semble se passer dans le second exemple ci-dessus, c'est que la séquence entre guillemets est à la fois employée et mentionnée, situation nécessairement exclue par Quine (cf. Garver 1965 : 232, qui remarque que la doctrine quinéenne présuppose que toute expression est soit employée, soit mentionnée, mais jamais les deux à la fois). Ce que réussissait ainsi à montrer Searle, c'est que la théorie du nom est inadaptée à toute une catégorie d'exemples pourtant attestés (ceux que, en 1997, Cappelen \& Lepore nommeront «mixed quotation»). Conclusion: soit on cherche une

\footnotetext{
15 D'autres auteurs ont fait semblable suggestion ironique : Davidson (1979: 30), Sørensen (1961: 189) et García-Carpintero (1994:255).

16 Nombreux sont les auteurs qui relèvent l'incompatibilité entre Théorie nominale et fonctionnement ordinairement conventionnel des noms (Garver 1965: 230-32, Christensen 1967 : 362 ; Davidson 1979 : 30 ; Bennett 1988 : 400-01; Washington 1992: 603 ; GarcíaCarpintero 1994: 254-55; Reimer 1996:137). La plupart d'entre eux voient là une raison suffisante pour rejeter la Théorie nominale.

${ }^{17}$ En français : L'intentionalité, trad. Claude Pichevin, Minuit, 1985.

${ }^{18}$ Nous ne tenons pas compte du cas non pertinent de l'ellipse : [John said he would call Abel, and] Gerald said he would [call] Henry.
} 
théorie unifiée et il faut alors rejeter la conception nominale. Soit on abandonne l'ambition de la théorie unifiée, mais il faut encore montrer que la Théorie nominale est valable dans son domaine propre.

\subsection{L'autonyme comme nom : riposte possible}

Il reste donc, à ce stade, une porte de sortie pour le tenant de la Théorie nominale. Il lui est loisible d'alléguer que sa théorie ne vise que les autonymes « purs », ceux dont on se sert lorsqu'on entend produire un énoncé délibérément métalinguistique, et que, de toute façon, l'idée de l'autonyme comme nom n'était pas à prendre au pied de la lettre, qu'il fallait seulement y voir une commodité de langage. Ce dernier argument n'est pas sans justification. Ainsi, plusieurs éléments donnent à penser que c'est bien une commodité de langage que les logiciens avaient en tête. Tout d'abord, souvenons-nous que Carnap n'a jamais utilisé de substantif Autonym, ce qui reflète la pensée que les autonymes ne sont pas des noms dans le lexique mais une variété exotique de "noms non lexicalisés » (l'expression est de Rey-Debove 1978 : 136). En second lieu, Quine aurait, selon Bennett (1988: 401), confié explicitement qu'il employait le verbe name dans le sens large de "désigner ». En troisième lieu, Tarski et Quine ont, parallèlement à la doctrine de l'autonyme comme nom, développé une théorie extensionnellement équivalente - que certains auteurs postérieurs nommeront «Théorie descriptive » de l'autonymie - qui voit dans l'autonyme l'abréviation d'une description telle que Le cinquième mot du poème "The Raven » ou encore d'une description « orthographique » comme Le mot composé de 'b', 'o', 's', 't', 'o', ' $n$ ', dans cet ordre. En plusieurs endroits, Quine et Tarski qualifient ces descriptions de noms (p.ex. Quine 1940: 26, 1974: 43 ; Tarski 1944 : 344, 1983 : 156-57) et il semble bien qu'ils fassent un usage interchangeable des termes de nom et description.

\subsection{Le postulat de l'expression monomorphémique}

On peut ainsi reprocher à certains critiques, notamment Searle et Recanati (1979), d'avoir "oublié », avec quelque opportunisme peut-être, que les philosophes logiciens qui ont proposé la Théorie nominale ne cherchaient pas à représenter de manière réaliste l'usage des langues naturelles. Il est néanmoins un précepte de cette théorie qui est entièrement assumé par ses défenseurs, et c'est un précepte qui pose des problèmes majeurs: "l'autonyme est un morphème unique » (Rey-Debove 1978: 149). Ce principe est défendu explicitement par Quine (même s'il n'utilise nulle part le mot morphème ou un de ses dérivés) :

Du point de vue de l'analyse logique chaque citation prise intégralement doit se concevoir comme étant un seul mot ou signe, dont les parties n'ont pas plus de 
valeur qu'un empattement de lettre ou une syllabe. [...] La signification du tout ne dépend pas de la signification des mots qui le constituent. (1940:26)

Ceci signifie que ce que Quine appelle l'« intérieur » des guillemets n'est pas en soi porteur de sens. Ainsi, le nom de personne Cicéron ne contribue pas davantage à la valeur sémantique de l'autonyme "Cicéron » que le verbe let ne contribue au sens de letter, le verbe (ou le nom) can à celui de canary ou le nom cat à celui de cattle (cf. Quine 1953 : 140, 1960 : 144). Il s'agit de concevoir la ressemblance entre Cicéron et "Cicéron» comme un "accident orthographique $» .^{19}$

On sent bien ici encore une intention prescriptive. Quine est parfaitement conscient de la dimension iconique de l'autonymie. Il utilise lui-même les termes picturing et hieroglyph. Mais c'est une dimension qui, puisqu'elle semble doter l'intérieur de la citation d'un potentiel signifiant, contrarie son projet de clarification, qui requiert que seule la citation entière apporte une contribution sémantique. Il faut donc apprécier l'axiome du morphème unique différemment de la décision de qualifier l'autonyme de nom. Si ce dernier choix n'est pas un enjeu théorique majeur, il en va tout autrement de la dimension monomorphémique. ${ }^{20}$ Cette dernière est entièrement assumée, et elle force Quine et les autres "nominalistes » à soutenir, avec une insistance qui peut paraître absurde, que la ressemblance systématique entre l'autonyme et l'expression qu'il mentionne est purement accidentelle, que les apparences sont trompeuses (cf. $1976: 161)$.

$\mathrm{Au}$ bout du compte, Quine préconisera l'usage des descriptions orthographiques (le mot composé de ' $b$ ', 'o', etc.) en remplacement des autonymes, précisément parce qu'elles sont dépourvues de toute dimension iconique : «c'est plutôt [la conception de] l'orthographe qui fournit la bonne

\footnotetext{
${ }^{19}$ Un des premiers critiques de Quine, Peter Geach, réagira précisément à la conception monomorphémique, qu'il jugeait tout à fait incapable de rendre compte du discours direct (que tous les philosophes s'accordent à traiter comme autonymique). En remplacement, il proposera une version "lexicale» de la Théorie descriptive, selon laquelle un autonyme de phrase est la somme des autonymes de mots qui composent la phrase. Ainsi, écrit Geach, « la citation " "man is mortal" " ne se comprend correctement que si nous lui attribuons la même signification qu'à “"man" \& "is" \& "mortal"” " (1957: 82).

Notons que personne ne nierait qu'il y a des différences entre l'autonyme de mots et le discours direct, ne fût-ce que celle-ci : la première est métalinguistique au sens strict, tandis que le second est «métadiscursif ». Mais ces deux catégories partagent également de nombreuses propriétés, tant du point de vue syntaxique que sémantique (les aspects pragmatiques sont, eux, nettement différents). Ces traits communs sont ce sur quoi les philosophes (et Rey-Debove) ont, à raison, mis l'accent, en tout cas dans un premier temps. Ceci n'est pas le signe d'une négligence ou d'une myopie, mais plutôt d'une bonne hiérarchisation des priorités : une théorie solide du noyau syntaxique et sémantique est utile à toute réflexion discursive, sociolinguistique, littéraire ultérieure.

${ }^{20}$ Ceux qui, comme Gómez-Torrente (2001 : 139), qui se réclame de Tarski, estiment que les critiques ont eu tort de prendre au pied de la lettre la terminologie du «nom », reconnaissent en revanche que la Théorie nominale soutient explicitement l'idée que l'autonyme n'est pas syntaxiquement complexe.
} 
analyse pour les besoins de la théorie logique des signes » $(1976: 241)$. On le voit, Quine s'occupe, ici comme ailleurs, de la clarté et du bon fonctionnement d'une langue adaptée à un usage scientifique. Mais, en l'occurrence, les décisions théoriques que motivent ses préoccupations rendent sa théorie inapte à expliquer l'autonymie dans les langues naturelles, puisqu'il se voit contraint d'en nier une caractéristique fondamentale : l'iconicité, comme rapport systématique entre expression mentionnante et mentionnée.

Pour clôturer cette discussion de la Théorie nominale, nous voudrions mettre en évidence une conséquence paradoxale des positions adoptées par Quine. Nous venons de voir que Quine fait de l'intérieur d'une citation un simple fragment de morphème, une suite accidentelle de lettres. D'un autre côté, pourtant, il affirme qu'un autonyme "dénote son intérieur» (1940:23). Prises ensemble, ces deux positions débouchent sur une situation absurde. Tout le monde s'accorde à dire que, lorsque nous utilisons un nom, disons Quine, c'est pour parler d'un individu, en l'occurrence Willard Quine. Mais ce n'est pas ce schéma que prédit la théorie quinéenne de la citation : il semble que lorsque j'utilise un autonyme pour parler d'un mot (de sa graphie, sa grammaire, son sens, son usage), je finis par parler en réalité d'un simple accident orthographique, puisque c'est apparemment cela que dénote mon autonyme. Or, un accident orthographique n'a certainement aucun sens, aucune grammaire, aucun usage.

Il faut en déduire que l'exposé de Quine est incohérent. Exit donc la Théorie nominale de l'autonymie.

\section{Quelle théorie pour remplacer la doctrine de l'autonyme comme nom ?}

Nous allons dans les pages qui suivent examiner trois théories qui ont été avancées comme substituts à la Théorie nominale et qui sont encore défendues actuellement. Mais avant de plonger dans le vif du sujet, nous voudrions évoquer un argument fréquemment invoqué dans les controverses entre philosophes du langage, l'argument de la simplicité. Parmi les reproches faits à la Théorie nominale, on lit souvent qu'elle donne une explication compliquée d'un phénomène parfaitement simple. Ainsi, Searle (1969:74), Recanati (1979 : 65ff), Davidson (1979 : 28), Washington (1992: 582-83) estiment que toute théorie qui dépeint l'autonymie comme quelque chose de mystérieux ou d'impénétrable ne peut qu'être fausse.

Il va de soi que les explications proposées en remplacement sont considérées, elles, comme étant beaucoup plus directes. Le problème est que tout le monde n'a pas la même notion de ce qui est simple. Ainsi, Davidson, persuadé qu'il est de la simplicité de sa "Théorie démonstrative» se voit pourtant rangé par Washington, adepte de la «Théorie de l'identité », parmi ceux qui se méfient des apparences et compliquent inutilement leur exposé. On remarquera, avec une petite pointe d'ironie, qu'il semblait à Tarski lui-même que sa théorie était « la plus naturelle et celle en accord le plus complet avec l'usage ordinaire des 
guillemets » (1983: 160), opinion que partage Gómez-Torrente, qui va même jusqu'à affirmer que « le côté naturel de la théorie tarskienne la rend préférable aux autres, qui sont en général plutôt exotiques et qui introduisent des complications qu'il s'agit, si possible, d'éviter » (2001 : 147).

On conclura que les professions de simplicité sont à prendre avec circonspection. Dans la suite, nous nous concentrerons plutôt sur trois autres critères utiles pour l'évaluation d'une théorie :

- elle doit être cohérente ;

- elle doit pouvoir rendre compte des données empiriques. Pour ce qui nous concerne ici, il s'agit de déterminer si la théorie est capable de justifier la grammaticalité/acceptabilité des phrases à dimension autonymique qui constituent son objet ;

- si plusieurs théories rendent adéquatement compte des données, la meilleure sera celle qui met le mieux en lumière les propriétés les plus significatives de son objet.

\section{Théorie de l'identité}

En même temps qu'il éreinte la Théorie nominale, Searle (1969) plaide pour qu'on lui substitue la conception moins «confuse» et moins «absurde » de l'identité. Searle n'en est pas l'inventeur, puisqu'elle remonte peut-être jusqu'aux logiciens stoïciens - cf. la thèse quelque peu équivoque de Chrysippe selon laquelle tout mot est par nature ambigu parce qu'il est aussi son propre nom (voir Atherton 1993: 38). De plus, cette théorie fut la plus courante chez les philosophes qui, de la fin du douzième siècle à la Renaissance, contribuèrent à la théorie de la "Supposition» (notamment Guillaume de Sherwood, Guillaume d'Ockham, Jean Buridan et Vincent Ferrier). Plus récemment, le philosophe danois Christensen a formulé des idées proches de celles de Searle avec quelques années d'avance.

L'idée centrale de la Théorie de l'identité, c'est que nous avons appelé autonyme jusqu'ici est en fait une expression qui est, dans un seul et même énoncé, à la fois employée (de manière déviante) et mentionnée. On se rappellera que nous soulignions chez Quine la parfaite compatibilité des notions d'emploi et de mention. Mais il ne faut pas négliger une différence de taille : chez Quine, l'expression employée et l'expression mentionnée sont deux expressions différentes (l'une guillemetée, l'autre pas). Selon la Théorie de l'identité, c'est une seule est même expression qui se trouve employée et mentionnée.

Cette théorie est très simple en apparence. En particulier, elle ne surcharge pas le lexique d'une infinité de noms propres métalinguistiques; elle soutient simplement que, dans

(5) Socrate est un philosophe passionnant,

(6) Socrate est un mot de sept lettres, 
c'est un token de la même expression-type qui apparaît en position sujet. La mention d'une expression n'entraîne jamais la création d'une expression d'un ordre supérieur, et l'autonymie n'est rien d'autre qu'un emploi particulier d'une expression tout à fait ordinaire.

Dans sa forme la plus élaborée (Washington 1992), la Théorie de l'identité se heurte à plusieurs objections sérieuses.

- Prenons l'idée de l'identité à la lettre. Si c'est la même expression qui mentionne et est mentionnée, alors, puisqu'un énoncé est une suite de tokens, l'autonyme ne peut lui aussi être qu'un token. Dès lors, seuls des tokens pourraient être mentionnés. Or, on voit bien que de nombreux autonymes réfèrent à un type, comme c'est le cas en (3) et (6). Plusieurs des autonymes en (1) désignent, eux aussi, des entités abstraites. Dans chacun de ces cas, le prédicat s'applique à une expression-type ou à une forme-type, pas à la seule occurrence qui figure dans l'énoncé. (Une objection de ce type apparaît chez García-Carpintero 1994 et Saka 1998.) Notons que, même si elle est puissante, cette objection n'est pas dirimante. On peut imaginer une réplique qui invoque une identité «type-type » plutôt que «token-token» - ces notions ont une existence indépendante en philosophie de l'esprit. A ma connaissance, pourtant, aucun théoricien de l'identité n'a fourni pareille réponse.

- Poursuivons l'idée que l'autonyme n'est qu'un emploi marqué de l'expression mentionnée. Ceci devrait entraîner que dans :

(7) Quoique est une conjonction,

c'est la conjonction de subordination quoique qui occupe la position sujet. Mais ceci nous forcerait à accepter que la grammaire du français contient l'une ou l'autre règle telle que

$$
\begin{aligned}
& \mathrm{P} \rightarrow \mathrm{Conj}+\mathrm{SV} \\
& \mathrm{SN} \rightarrow \mathrm{Conj}
\end{aligned}
$$

Autrement dit, soit on réécrit une phrase P comme la suite d'une conjonction et d'un syntagme verbal, soit on réécrit un syntagme nominal (occupant standard de la position sujet) comme conjonction. Si l'on songe que des mots de n'importe quelle catégorie grammaticale peuvent être mentionnés - il suffit qu'ils soient le bon argument d'un prédicat adéquat (métalinguistique ou neutre) -, on voit à quel foisonnement de règles nouvelles on s'expose. Ceci aura pour conséquence une grammaire trop puissante, capable d'engendrer une multitude d'énoncés mal formés, comme Avant que va à la plage ou Jamais est prêt. (On trouve une objection de ce type chez Sørensen 1961 et Reimer 1996.) 
- Il est un fait que n'importe quelle séquence de sons ou de lettres peut devenir autonyme. Ainsi, les énoncés suivants sont parfaitement bien formés et interprétables :

(8) «Rlkhfg LFHCAlmhhlcleoooooo » ne veut rien dire du tout.

(9) Elle me fit « ... ?! ... !!!!» et je pris mes jambes à mon cou.

On est en droit de se demander ce que peut bien signifier ici l'idée que c'est la même expression qui mentionne et est mentionnée, puisque la séquence mentionnante n'est pas une expression. Comment l'autonymie peut-elle se concevoir ici comme un emploi particulier d'un «machin » qui ne connaît pas d'autre emploi linguistique par ailleurs?

Ces trois objections sont sérieuses et peut-être condamnent-elles définitivement la Théorie de l'identité, en tout cas dans une lecture radicale mais, sinon, à quoi bon invoquer l'identité ? De fait, il existe d'autres objections encore ; le lecteur intéressé pourra consulter Saka (1998 : 122-25).

\section{Théorie démonstrative}

On en attribue généralement la paternité à Donald Davidson (1979). S'il est vrai que c'est l'article de Davidson qui a popularisé la Théorie démonstrative, cette attribution constitue néanmoins une injustice historique, puisque le linguiste danois Sørensen en avait énoncé les principes dès 1958, et notamment l'idée audacieuse qu'une «paire de guillemets est un pronom démonstratif métalinguistique » (1958: 51). Par ailleurs, François Recanati (2001 : 653) cite un texte d'Arthur Prior datant de 1971 qui avance exactement le même argument.

C'est pourtant Davidson qui sera l'influence majeure sur les nombreux philosophes qui reprendront (certains aspects de) sa Théorie démonstrative, citons Goldstein (1984), Bennett (1988), Reimer (1996), Seymour (1996), Cappelen \& Lepore (1997). En gros, tous les principes importants de la théorie davidsonienne découlent de l'hypothèse que les guillemets sont un pronom démonstratif. Nous illustrons. La « forme logique » d'un exemple comme (3) est en réalité quelque chose comme :

(FL-3) Boston. Ceci est un mot de deux syllabes.

Comme l'écrit Davidson, il faut « imagine[r] que l'occurrence de «ceci» soit complétée par un index qui pointe vers l'occurrence de [«Boston»]» (1979: 38). Ce qui est frappant, c'est que l'autonyme lui-même ne fait pas partie de la forme logique de (3). Si, avec Davidson, on considère que la forme logique reflète aussi l'analyse syntaxique de l'énoncé, on est amené à conclure que l'autonyme ne contribue ni à la structure grammaticale, ni à l'interprétation 
sémantique de la phrase citante. Il n'est qu'un élément du contexte, comme le serait tout référent attribuable à un syntagme contenant un démonstratif !

Cette propriété, que Davidson qualifie d' " inertie » sémantique et syntaxique de l'autonyme, est rendue nécessaire par le fait que l'on est autorisé à citer « n'importe quoi », y compris des suites agrammaticales et dépourvues de sens. Nous répétons (8) :

(8) « Rlkhfg LFHCAlmhhlcleoooooo » ne veut rien dire du tout.

Si l'intérieur de la citation contribuait à la syntaxe et à la sémantique de (8), on serait amené à conclure que (8) est une phrase agrammaticale et dépourvue de contenu sémantique. Or, nos intuitions nous portent à penser, au contraire, que (8) est à la fois bien formée et pleine de sens. Une analyse semblable peut s'appliquer aux exemples (1-i) et (9), qui sont similaires. On voit que sur ce point la Théorie démonstrative fait mieux que celle de l'identité. ${ }^{21}$

Qu'en est-il maintenant d'un exemple comme (7) ? Jusqu'ici, à l'instar des auteurs dont nous traitons, nous n'avons prêté attention qu'aux guillemets. Mais il existe d'autres manières de marquer l'autonymie, notamment par l'emploi des italiques. La théorie davidsonienne peut-elle en rendre compte ? Certains auteurs pensent que non. Ainsi, Reimer (1996 : 135) estime qu'il n'est pas raisonnable de considérer que les italiques sont un morphème démonstratif alors qu'en même temps la séquence citée est privée de fonction sémantique et syntaxique. Les démonstrativistes n'ont, à ma connaissance, pas fourni de réponse circonstanciée à cette objection, se contentant, comme Lepore (1999: 691), de supposer que les italiques et autres marques possibles se comportent de manière semblable aux guillemets. Le seul à avoir abordé la question de front est le pré-davidsonien Sørensen, qui n'hésitait pas à écrire que « le sujet de discours est la séquence en italiques, et le sujet [grammatical] les italiques. Le sujet, dans le cas des italiques, peut [...] être qualifié de suprasegmental » (1961 : 187).

Si l'on accepte la justification aventureuse de Sørensen, on peut alors avancer que des exemples comme (7) ne posent aucun problème au démonstrativiste, puisque sa théorie empêche la catégorie grammaticale de l'expression citée d'interférer avec la syntaxe de la phrase citante. Encore un point à l'actif des davidsoniens.

Mais il est temps de passer aux objections, qui, on va le voir, sont accablantes. ${ }^{22}$

- Une faiblesse de la Théorie démonstrative provient de l'importance qu'elle accorde aux guillemets (tout comme les Théories nominale et descriptive). On vient de voir le problème que posaient les italiques, mais qu'en est-il des cas où

\footnotetext{
${ }^{21}$ L'inertie syntaxico-sémantique de la séquence intérieure aux guillemets joue ici exactement le même rôle que l'axiome du morphème unique chez les tenants de la Théorie nominale.

${ }^{22}$ On trouve de nombreuses critiques dans la littérature, certaines desquelles seront reprises dans notre discussion. Pour plus de détails, voir Washington (1992), Reimer (1996, 2005), Saka (1998), Tsohatzidis (1998, 2005), Recanati (2001).
} 
l'autonyme n'est pas signalé par un signe typographique ou de ponctuation ? Si l'intérieur de l'autonyme est inerte, et seuls les guillemets (ou les italiques) remplissent une fonction grammaticale, comment rendre compte d'exemples comme (4) (Boston is disyllabic) qui, n'en déplaise à Quine, sont monnaie courante ? Une possibilité est de dire qu'ils sont simplement mal formés. Dès lors, la théorie n'a pas en s'en occuper. C'est une tentation qui travaille Cappelen \& Lepore puisque, dans une critique de Saka (1998), ils soutiennent qu'il doit encore être «établi que pareille mention sans guillemets existe » (1999 : 746). Toutefois, cette voie est stérile, étant donné la profusion d'autonymes non marqués à l'écrit (cf Rey-Debove 1978 : 69-71), mais aussi à l'oral (situation que presque aucun philosophe jamais n'aborde), qui ne semblent poser aucun problème d'acceptabilité chez les scripteurs-locuteurs non philosophes.

Une autre possibilité consiste à dire que l'absence de marques résulte d'une ellipse. Mais, rétorque Washington (1992:589-90), cette stratégie échoue en tout cas pour les énoncés oraux qui ne marquent pas l'autonyme. Ainsi, de "Bonjour » est une salutation, on peut dériver :

(10) «...» est une salutation,

énoncé acceptable selon Davidson et ses partisans, puisque les guillemets sont un morphème démonstratif. Mais, d'une énonciation orale de Bonjour est une salutation, on ne peut dériver que :

(10’) est une salutation,

qui est syntaxiquement mal formé. Washington ajoute que, à sa connaissance, aucune théorie linguistique n'autorise une ellipse du sujet dans un cas comme (10'). Conclusion : la Théorie démonstrative est fausse. Il faut toutefois noter que Manuel García-Carpintero a tenté de montrer qu'on pouvait contrer l'objection de Washington en invoquant des indices contextuels ou des implicatures conversationnelles. Le lecteur intéressé consultera GarcíaCarpintero (1994 : 262-63).

— La deuxième objection repose sur l'existence d'exemples comme :

(11) Gerald said he would 'consider running for the Presidency',

que nous avons déjà utilisé pour pointer les lacunes de la Théorie nominale. A première vue, ces énoncés posent ici le même genre de problème. En effet, si l'on prend au pied de la lettre l'affirmation que les guillemets sont un morphème démonstratif, alors on devrait pouvoir remplacer la citation en (11) par this, ce qui produit un énoncé mal formé :

(FL-11) * Consider running for the Presidency. Gerald said he would this. 
L'écueil est sérieux, mais certains démonstrativistes y ont apporté une réponse. J'y reviendrai en détail à la section 8 , qui est consacrée aux énoncés comportant une séquence simultanément employée et mentionnée.

- La troisième objection que nous souhaitons développer n'est mentionnée nulle part dans la littérature, mais elle vaut néanmoins d'être discutée. Elle s'appuie sur une variété intéressante mais fort négligée de l'autonymie. ${ }^{23}$ Il est des cas où l'autonyme ne fonctionne pas comme un syntagme nominal, mais comme la tête d'un SN métalinguistique. Voici deux exemples :

(12) $[\ldots]$ en prononçant cet on, Marat regarda Danton. (Victor Hugo, cité dans Rey-Debove $1978: 66$ )

(13) 'My mother teaches, sir,' I said, liking the 'sir' and liking the fact that Sir Charles liked it. (Stephen Fry, The Stars' Tennis Balls, Londres, Arrow books, $2001: 66$ )

Comme dans le cas de la seconde objection, le remplacement de la séquence guillemetée par un démonstratif donne un énoncé mal formé : (Les formes logiques ci-dessous sont adaptées de manière « charitable ».)

(FL-12) * On. En prononçant ce ceci, Marat regarda Danton.

(FL-13) * Sir. I liked the this and liked the fact that Sir Charles liked it.

Mais, dans ce cas-ci, il ne semble pas raisonnable d'espérer trouver une solution, comme pour les « hybrides » tels que (11). La raison en est que les autonymes en (12) et (13) ne sont pas utilisés référentiellement, même s'ils font partie d'un SN qui réfère. La présence d'un déterminant (cet et the) qui a déjà pour rôle d'indiquer la dimension référentielle du $\mathrm{SN}$ entier, semble bien exclure que, au sein même du $\mathrm{SN}$, un autre morphème - les guillemets - aient aussi une référence (différente de celle du $\mathrm{SN}$ entier?).

\section{Théorie de l'illustration}

Les trois théories que nous avons passée en revue racontent chacune une histoire différente sur le moyen par lequel un autonyme mentionne son référent. Selon la Théorie nominale, tout autonyme est une sorte de nom propre composé d'une paire de guillemets ceignant une suite de lettres. Cet intérieur de guillemets ne joue aucun rôle sémantique, puisque c'est le nom entier (morphème unique) qui réfère. (Le cas oral n'est pas envisagé.) Selon la Théorie démonstrative, ce sont les guillemets seuls qui réfèrent. Comme dans la Théorie nominale, l'intérieur n'a d'activité ni syntaxique ni sémantique dans la phrase citante. (L'autonyme à

${ }^{23}$ La principale exception est, une fois encore, Rey-Debove. 
l'oral, lorsqu'il est pris en compte, est traité comme un cas d'ellipse des guillemets.) Enfin, selon la Théorie de l'identité, c'est l'intérieur même (lorsqu'il $\mathrm{y}$ a des guillemets) qui réfère (à lui-même). (Le cas oral ne pose pas de problème puisque la théorie n'impose aucun rôle sémantique aux guillemets.)

On peut penser que ces trois théories épuisent les combinaisons concevables (guillemets + intérieur; guillemets seuls ; intérieur seul). Or, il reste une autre possibilité, développée par François Recanati dans un article de 2001, et qui consiste à dire que c'est par un " acte d'illustration » que le locuteur qui emploie un autonyme parvient à référer. Recanati s'appuie sur un article des psychologues Herbert Clark et Richard Gerrig (1990) dans lequel ils jetaient les bases d'une théorie de la citation comme demonstration, terme qu'ils entendaient au sens d'«illustration par l'exemplification ». ${ }^{24}$ Là où les davidsoniens insistaient sur la dimension indexicale de l'autonymie, Clark \& Gerrig, puis Recanati, en soulignent la dimension iconique.

Le premier intérêt de la théorie de l'illustration, c'est qu'elle n'est pas uniquement une théorie de la citation. Elle s'applique à d'autres sortes d'énoncés à forte composante iconique, y compris des choses peut-être curieuses, mais parfaitement communes, comme :

(14) J'ai appelé [GESTES, GRIMACES ET BORBORYGMES DESIGNANT « CET IMBECILE DE JULES »] et il m'a raconté les mêmes salades que l'autre fois.

(15) Le type s'est mis à gueuler et nous on a [GESTICULATIONS IMITANT LA FUITE A LA COURSE], tu vois.

Dans ces exemples, des gestes couplés à des expressions faciales remplissent une fonction syntaxique et sémantique habituellement dévolue à des constituants linguistiques.

L'idée maîtresse de Recanati est que, dans l'autonymie, c'est non pas au moyen des mots ou des marques linguistiques en tant que tels que le locuteur réfère, mais par l'acte d'illustration qu'il accomplit. C'est cet acte qui se voit « recruté linguistiquement », comme SN ou autre, et non les mots ou les marques qui servent à son accomplissement. La preuve en est que l'acte d'illustration ne nécessite aucune production de mots, comme nous l'avons constaté avec les exemples (14) et (15). On voit comment cette intuition permet à Recanati d'éviter l'impasse à laquelle semblait mener l'échec relatif des trois autres théories. Nul besoin ici d'attribuer une capacité référentielle aux guillemets, ou à l'intérieur des guillemets, ou à la séquence constituée des deux.

\footnotetext{
${ }^{24}$ Nous avons choisi de rendre l'anglais demonstration par illustration, jugeant que le terme de démonstration risquait d'embrouiller le lecteur francophone. D'autres traductions, comme monstration et ostension, ont été rejetées parce qu'elles suggèrent que l'autonyme a un fonctionnement indexical (comme les démonstratifs) et non iconique.
} 
L'avantage de la théorie de Recanati, c'est qu'elle regroupe les points forts des théories précédentes sans hériter des objections qu'elles soulèvent. Ainsi, l'idée du recrutement syntaxique de l'acte d'illustration rend compte de l'intuition fondatrice de la Théorie nominale : que les autonymes fonctionnent d'ordinaire comme des SN. Par ailleurs, comme dans la Théorie démonstrative, l' "intérieur» de l'autonyme n'est pas actif du point de vue syntaxique et sémantique dans la phrase citante, puisque c'est l'acte d'illustration qui y joue ces rôles. Cependant, Recanati ne se retrouve pas contraint à faire reposer tout l'édifice de sa théorie sur des marques dont les données empiriques nous indiquent qu'elles sont souvent absentes. Ce dernier point va dans le sens de la Théorie de l'identité, qui traite les guillemets comme une marque facultative de ponctuation.

Enfin, l'exposé de Recanati possède un dernier avantage notable sur les théories nominale et démonstrative. Il ne s'y trouve aucune obligation d'assigner une fonction référentielle à toutes les citations, puisque le recrutement syntaxique n'est pas constitutif de l'acte d'illustration. Lorsque le recrutement a lieu, Recanati parle de «citation fermée ». Lorsqu'il n'a pas lieu, il parle de « citation ouverte ». C'est ainsi que, dans des exemples comme (11) ou :

(16) A l'issue du verdict, la famille de François C. a tenu à déclarer, via leur avocate, qu'ils "laissaient la porte ouverte " [...]. (Le Monde, $11 / 10 / 2004$; édition en ligne),

la théorie n'impose pas de la présence d'un syntagme nominal capable de référer, présence syntaxiquement indésirable puisqu'elle mène droit à la conclusion que l'énoncé est agrammatical. ${ }^{25}$

\section{Les hybrides}

On a vu plus haut que les cas où la séquence mentionnée se révèle être simultanément employée posaient des problèmes tant à la Théorie démonstrative qu'à la Théorie nominale (mais pas à la Théorie de l'identité). Il est temps maintenant de nous pencher plus longuement sur ce phénomène, dont la caractéristique centrale semble tenir au fait que l'énoncé où il se produit préserverait son contenu sémantique et sa grammaticalité même s'il n'incluait plus aucune dimension autonymique. Nous illustrons brièvement. De (16), on passe à :

\footnotetext{
${ }^{25}$ La théorie de Recanati est beaucoup plus fouillée que cet exposé sommaire pourrait le laisser croire. Nous aurons heureusement l'occasion d'éclairer davantage ses idées sur la citation ouverte dans la section suivante. Remarquons qu'elle a fait l'objet de plusieurs critiques. Voir les articles de Benbaji, Cappelen \& Lepore, García-Carpintero, Gómez-Torrente et Saka dans le ${ }^{\circ}$ 17 du Belgian Journal of Linguistics (2005).
} 
(16') A l'issue du verdict, la famille de François C. a tenu à déclarer, via leur avocate, qu'ils laissaient la porte ouverte. ${ }^{26}$

Nous utilisons ici les guillemets/leur absence pour expliciter une intention de mention/l'absence d'une telle intention. Nous voudrions toutefois souligner que nous n'estimons pas que les guillemets (ou d'autres marques) soient nécessaires à l'accomplissement d'une intention de mention. Sinon, nous serions forcé d'accepter qu'il ne peut y avoir mention sans marquage particulier, ce qui va à l'encontre de toutes les observations empiriques (voir supra).

Ceci étant clarifié, on voit que si l'on supprime l'intention de mention qui, parmi d'autres, sous-tend l'énonciation de (16), on obtient un énoncé (16') tout à fait complet et bien formé du point de vue sémantique et syntaxique. En revanche, si l'on effectuait la même opération sur (17) ou (18), qui contiennent des autonymes « purs », on produirait :

(17) * Elle a hurlé pas avec ta mère, et pas avant Noël!.

(18) * Bonne femme est laid et insultant. ${ }^{27}$

Autrement dit, sans intention de mention, (17) et (18) sont des énoncés agrammaticaux et à la limite de l'interprétabilité. Et, même dans les rares cas où pareils énoncés seraient interprétables, leur contenu sémantique serait radicalement différent de celui de l'énoncé comportant un autonyme (p.ex. Ce "petit philosophe de merde » est bien peu respectueux vs. Ce petit philosophe de merde est bien peu respectueux).

On le voit, la distinction fondamentale entre les cas d'autonymie standard et les cas hybrides comme (11) et (16), c'est que l'intention de mention est constitutive des premiers (au sens où elle est nécessaire à leur acceptabilité sémantique et syntaxique) mais pas des seconds.

Les types d'exemples dont il va être question dans la présente section ont longtemps échappé à l'attention des philosophes du langage. On ne s'étonnera pas qu'ils aient été négligés par les fondateurs de la discipline, tout préoccupés qu'ils étaient par des langages formalisés qui excluaient, par définition, de tels énoncés hybrides. On ne s'étonnera pas non plus que ce soient des linguistes qui aient été les premiers à les mettre en évidence. Ainsi, dès 1967, la linguiste polonaise Mayenowa étudiait des exemples comme :

\footnotetext{
${ }^{26}$ Certains francophones ont le sentiment qu'un verbe comme déclarer contraint davantage la forme d'un rapport de discours indirect que dire, par exemple. En d'autres termes, lorsque déclarer introduit du discours indirect, ces locuteurs se permettent moins de libertés dans le choix des mots.

27 J'insiste sur le fait que ces énoncés doivent être lus comme dépourvus non seulement de guillemets mais de toute dimension autonymique.
} 
(19) Certains enfants gardent assez longtemps la tendance à estropier les mots en «mangeant » des sons entiers. (1967: 1317),

exemples dont elle suggérait que leur «message ne peut être exprimé que par deux propositions conjointes, dont l'une relève de la langue des choses, et l'autre, de la métalangue » (1967: 1317). L'esprit de cette analyse est d'ailleurs très proche de celle que donnent de nombreux philosophes aujourd'hui encore (p.ex. Predelli 2003, Gómez-Torrente 2005, Potts 2004). Après Mayenowa, le jalon le plus important est la publication du Métalangage en 1978. Rey-Debove $\mathrm{y}$ introduit la notion de «connotation autonymique», une configuration langagière qui participe à la fois de la connotation et du métalangage : « c'est un système qui dénote « le monde » (signifié dénotatif) et connote les signes utilisés pour en parler (signifié connotatif)» (1997a: 342). Cette notion recouvre tous les exemples que nous traiterons comme hybrides dans la présente section. ${ }^{28}$

\subsection{Citation mixte}

À ma connaissance, le premier philosophe à avoir signalé la question des hybrides est Donald Davidson. Dans son article de 1979, il se confronte à l'exemple suivant :

(20) Quine says that quotation “... has a certain anomalous feature." $(1979: 28)$

Davidson soutient que de tels exemples peuvent être aisément expliqués par la Théorie démonstrative. Son argument est simple - mais peu convaincant : ce que Davidson a affirmé auparavant - que seuls les guillemets réfèrent et font partie intégrante de la phrase - était trop radical. Puisqu'un pointeur (ici, les guillemets) est capable d'indiquer tout ce qui se trouve sous sa portée, il arrive qu'il pointe également vers « une inscription en emploi actif» (1979:39). Si l'on peut être d'accord avec la description que donne Davidson (emploi et mention simultanés), on ne peut s'empêcher de relever que l'analyse proposée est incompatible avec l'idée que les guillemets sont un pronom démonstratif. Si c'est bien ce qu'ils sont, alors, ils occupent la position syntaxique d'un SN, comme on l'a vu dans la seconde objection présentée dans la section 6 .

En 1997, Cappelen \& Lepore (désormais, $C \& L$ ) publient un article qui fera pas mal de bruit dans le petit monde des théoriciens de la citation. Ils

\footnotetext{
${ }^{28}$ Pour être complet, la connotation autonymique s'étend à des exemples comme Giorgione was so called because of his size, qui ne sont pas hybrides, parce qu'ils ne contiennent aucun segment qui soit à la fois employé et mentionné : Giorgione est en emploi ordinaire, et c'est so (anaphorique ou déictique) qui réfère au mot Giorgione. Mais so n'est pas autonyme ; il est au contraire hétéronyme... ce qui nous fait penser que Rey-Debove a tort de voir dans cet énoncé une illustration de la connotation autonymique. C'est hélas une question que nous n'avons pas la place de développer ici.
} 
ambitionnent d'y exposer la première théorie « unifiée » de la citation pure, des discours direct et indirect, ainsi que de l'hybride de ces derniers, qu'ils nomment " mixed quotation $»{ }^{29}$ Leur théorie se veut une extension du cadre proposé par Davidson. Nous donnons ici des exemples de discours direct, discours indirect et citation mixte, ainsi que leur forme logique selon C\&L (1997) :

(21) Alice said "Life is difficult to understand".

(22) Alice said that life is difficult to understand.

(23) Alice said that life "is difficult to understand".

(FL-21) $\exists u(\operatorname{Said}(\mathrm{a}, u) \& \mathrm{ST}(u$, these)). Life is difficult to understand

(FL-22) $\exists u(\operatorname{Said}(\mathrm{a}, u) \& \mathrm{SS}(u$, that)). Life is difficult to understand

(FL-23) $\exists u(\operatorname{Said}(\mathrm{a}, u) \& \mathrm{SS}(u$, that) \& $\mathrm{ST}(u$, these $))$. Life is difficult to understand

Pour déchiffrer les formes logiques, il faut savoir que $u$ est une variable d'énoncé ( « utterance »), ST un prédicat (« sametokens ») qui désigne, en gros, la relation qu'entretiennent deux tokens lorsqu'ils sont des occurrences du même type (type qui doit être déterminé contextuellement), et $S S$ un prédicat («samesays ») qui désigne, en gros, la relation qui lie deux énoncés lorsqu'ils expriment la même proposition. Comme on le voit, la forme logique de la citation mixte (FL-23) combine les formes logiques des discours direct et indirect. Il convient toutefois de préciser que les deux démonstratifs qu'elle contient n'ont pas la même portée : that réfère à Life is difficult to understand et these seulement à is difficult to understand.

De cette manière, en proposant une explication «quantificationnelle » de la citation, C\&L offrent une réponse à la seconde objection de la section 6 - le fait que la théorie démonstrative originelle prédit, à tort, que toute séquence entre guillemets doit pouvoir être remplacée par un pronom démonstratif. De plus, ils formalisent l'idée, pour eux fondamentale, que les guillemets dans la citation mixte jouent un rôle sémantique puisqu'ils modifient les conditions de vérité de l'énoncé. En effet, la forme logique (FL-23) détermine des conditions de vérité qui requièrent non seulement que les paroles prononcées par Alice expriment la même proposition que Life is difficult to understand mais aussi que ces paroles soient un token du même type que celui auquel renvoie l'occurrence de is difficult to understand. Dans l'interprétation la plus évidente, ${ }^{30}$ ce dernier point signifie que, si Alice n'a pas prononcé, verbatim, les paroles is difficult to understand, alors (23) est faux.

\footnotetext{
${ }^{29}$ C\&L démontrent ainsi leur ignorance des travaux de Rey-Debove. Moralité : si l'on veut être lu des philosophes du langage, il vaut mieux s'exprimer en anglais et être philosophe analytique ou linguiste formel.

${ }^{30} \mathrm{Je}$ ne discute pas la possibilité qu'Alice se soit exprimée dans une autre langue que l'anglais, ou soit bègue et ait répété dix fois la même syllabe. Le terme verbatim pose quantité de problèmes qui retiennent enfin l'attention des philosophes. (Cf. Saka 2005 et Tsohatzidis 2005).
} 
L'approche de C\&L a été accueillie avec enthousiasme par certains (Benbaji, García-Carpintero, Simchen) et critiquée sévèrement par d'autres (Recanati, Reimer 2005, Saka 2005, Tsohatzidis). Un des enjeux majeurs du débat consiste à déterminer si les guillemets (ou autres marques de la citation) ont une valeur sémantique. Tous les auteurs actuels qui se réclament de Davidson répondent « oui » à cette question. En revanche, ceux qui rejettent le principe fondateur de la Théorie démonstrative - les guillemets comme pronom démonstratif répondent « non ».

Le débat est toutefois plus complexe qu'on pourrait le supposer. Les tenants d'une explication pragmatique des guillemets sont partagés (notamment) sur la question de savoir si le refus de leur attribuer une valeur sémantique doit entraîner un refus de leur accorder un impact vériconditionnel. Je n'ai pas ici la place pour développer les différentes versions de l'explication pragmatique. ${ }^{31} \mathrm{Je}$ me contenterai d'esquisser deux réponses, celles de Stainton (1999) et de Recanati (2001).

Pour rendre compte de nos intuitions concernant des exemples comme (20) ou (23), Stainton (1999: 273-74) en appelle à la distinction entre vérité et àpropos (felicity). Stainton admet que si Quine a en réalité utilisé les mots has some unusual feature, et si Alice a dit is tough to understand, on pourra avoir l'impression que (20) et (23) sont faux. Mais, dit Stainton, c'est là confondre la vérité et l'à-propos, confusion dont se rendent coupables C\&L. La bonne explication consiste à reconnaître que (20) et (23) sont utilisés mal à-propos (infelicitously) si les citations ne rendent pas les paroles réellement prononcées par Quine et Alice, mais ceci ne signifie pas que (20) et (23) seraient alors faux. Pour Stainton, le choix des mots entre guillemets n'est pas différent du volume, de l'intonation, de la vitesse d'élocution. Personne ne jugera que mon énonciation de (20) ou (23) est fausse si je prononce en bégayant et à bas volume les mots entre guillemets, alors que Quine et Alice les auraient énoncés avec fluidité et très fort. Ma présentation des paroles de Quine et d'Alice peut être tenue pour malheureuse ou trompeuse, mais pas pour fausse. Le même raisonnement s'applique au choix des mots. Conclusion : les guillemets n'ont pas d'impact sur les conditions de vérité.

La stratégie adoptée par Recanati est différente. Elle consiste essentiellement à établir qu'il existe une distinction entre les conditions de vérité « compositionnelles» et « intuitives» d'un énoncé. Les premières (c-content) correspondent à l'usage traditionnel en logique et philosophie du langage : elles sont purement « sémantiques ». Les secondes (i-content), selon Recanati, rendent réalistement compte de ce qu'un locuteur habile tiendrait pour vrai ou pour faux : elles incluent une dimension "pragmatique ». Un petit exemple. Si, à un enfant qui, s'étant éraflé le genou, s'est mis à hurler, je dis :

(24) Oh, tu ne vas pas mourir!,

${ }^{31}$ Reimer (2005) constitue une excellente introduction sur ce point. 
tout locuteur francophone comprendra que j'ai voulu dire « tu ne vas pas mourir de cette égratignure ». C'est pourquoi il tiendra l'énoncé (24) pour vrai dans ce contexte. Ceci va à l'encontre de l'analyse traditionnelle, qui attribuera la forme logique :

(FL-24) $\exists x$ (Destinataire(x) \& $\neg \operatorname{Mortel}(\mathrm{x}))[=$ Il existe un individu qui est le destinataire de l'énoncé et qui n'est pas mortel] $]^{32}$

à l'énoncé et l'évaluera comme faux puisqu'on sait que « tous les hommes sont mortels ».

La distinction qu'établit Recanati a l'avantage de s'appliquer à une grande variété de cas - les énoncés où l'on identifie des " constituants non articulés » qui entraînent un " enrichissement pragmatique » des conditions de vérité ; on ne peut donc lui reprocher d'être « ad hoc ». Comment l'utilise-t-il en rapport avec la citation mixte? L'idée est simple. Reprenons (20) et comparons-le à sa variante sans citation :

(20) Quine says that quotation “... has a certain anomalous feature."

(20') Quine says that quotation has a certain anomalous feature.

Pour Recanati, les deux énoncés ont le même contenu compositionnel. Les guillemets n'ont donc pas d'impact sémantique. En revanche, le contenu intuitif de (20) est plus riche que celui de (20'), puisqu'il comporte le réquisit que Quine ait employé les mots has a certain anomalous feature: (20) implique sémantiquement (20'). Il y a, comme en (24), enrichissement des conditions de vérité.

La distinction que fait Recanati lui permet de :

(i) dire, contre C\&L, que les guillemets ne modifient pas la sémantique de l'énoncé ;

(ii) montrer qu'il y a bien un effet vériconditionnel de l'usage des guillemets dans la citation mixte ;

(iii) ne pas devoir nier, comme le fait Stainton, l'intuition de cet effet vériconditionnel.

Le stratégies de Stainton et de Recanati ont chacune leur attrait, mais il reste des questions auxquelles elles doivent encore répondre. Nous n'aurons pas le temps de développer ces questions ici. Nous nous contenterons de dire que la proposition de Recanati a séduit une série de philosophes et linguistes parmi lesquels Abbott, Reimer et nous-même.

\footnotetext{
${ }^{32}$ Nous avons simplifié la forme logique en n'y faisant pas apparaitre une variable pour l'énoncé lui-même.
} 


\subsection{Scare quoting ${ }^{33}$}

Si l'intérêt des philosophes pour la citation mixte est assez récent, la réflexion sur le scare quoting l'est encore davantage. (Ici aussi Mayenowa et, surtout, ReyDebove font figure de précurseurs). Ainsi, les exemples d'hybrides chez Davidson, Searle et C\&L appartiennent tous à la catégorie de la citation mixte. De fait, pour les démonstrativistes, et particulièrement pour $C \& L$, qui prétendent à une théorie unifiée des variétés de la citation, le scare quoting pose un problème de taille. En effet, pour introduire le scare quoting, il n'y a pas de verbe de parole (say) qui puisse s'interpréter comme subsumant les sens combinés de sametokening et samesaying. C'est pourquoi, C\&L sont dans l'impossibilité de proposer une explication s'appuyant sur l'idée de deux propositions conjointes, l'une mondaine, l'autre métalinguistique, qui se reflèterait dans une forme logique semblable à celle de la citation mixte, pour des exemples tels que (19) ou (25) :

(25) Le gouvernement a encore augmenté le budget du Ministère de la « défense ».

Dans un premier temps, C\&L ont adopté la position suivante : le scare quoting était « peut-être une autre variété de la citation et [valait] certainement la peine d'être discuté » et présentait «d'importantes similitudes » (1997:430, note 2) avec la citation mixte, mais ils ne disposaient pas de la place nécessaire pour l'intégrer à leur article. Actuellement (C\&L 2005), ils reconnaissent que le scare quoting est redevable à une autre théorie que les autres variétés de la citation. Dès lors, il semble que, leur théorie « unifiée » ne pouvant être étendue au scare quoting, C\&L aient été acculés à admettre que les guillemets sont ambigus. Pourtant, ils récusent l'idée de l'ambiguïté des guillemets, ${ }^{34}$ pour des raisons, subtiles, que nous n'avons pas l'occasion d'examiner ici et qui ne convainquent pas tous leurs lecteurs. Seul parmi les adeptes d'une théorie sémantique des hybrides, Mario Gómez-Torrente, qui se réclame non de Davidson mais de Tarski, embrasse sans retenue l'option de l'ambiguïté des guillemets.

$\mathrm{Si}$, maintenant, l'on se tourne vers les partisans d'une explication pragmatique, il est clair qu'ils ont moins de problèmes à rendre compte du scare quoting. Les diverses implications que l'usage "distanciateur» des guillemets peut servir à communiquer seront traitées comme des effets contextuels, qui viennent en complément du contenu sémantique de l'énoncé.

\footnotetext{
33 J'utiliserai le terme anglais, parce que le terme français qui s'en rapproche le plus (« îlot textuel ») désigne aussi (et avant tout) les éléments mis entre guillemets dans un discours indirect, autrement dit la citation mixte.

${ }^{34}$ Tout comme Benbaji, qui lui aussi se réclame de Davidson et donne un traitement séparé de la citation mixte et du scare quoting.
} 
Il est toutefois une catégorie redoutable, que Recanati range sous l'étiquette d' « emplois non-cumulatifs » (2001: 670-80). Ces emplois se divisent en trois cas : celui du «changement de langue» («language-shift»); celui du « changement de circonstance d'évaluation» ( « circumstance-shift»); celui du « changement de situation» («situation-shift»). Ces trois notions sont ellesmêmes tributaires d'un cadre théorique qui postule qu'un contexte $k$ « est un triple $\langle L, s, c\rangle$ où $L$ est une langue, $s$ une situation d'énonciation comprenant un certain nombre de paramètres [essentiellement, les temps et lieu de l'énonciation, l'énonciateur, le destinataire ; c'est-à-dire les éléments qui permettent d'assigner une valeur sémantique aux expressions indexicales ou déictiques], et $c$ une circonstance d'évaluation [un monde possible par rapport auquel une valeur de vérité est attribuée à l'énoncé] » (2001: 679).

Nous n'allons pas pouvoir faire davantage qu'illustrer les problèmes que soulèvent les variétés non-cumulatives du scare quoting. Mais cette présentation a son intérêt, parce qu'elle permet de prendre la mesure du chemin parcouru en un peu plus d'un siècle de réflexion sur l'autonymie d'abord, puis sur les phénomènes hybrides à dimension autonymique.

L'exemple qui suit comporte un «changement de langue ». Imaginons que Nadia et moi sachions que Jules pense que M. Dupont s'appelle « M. Dulong ». Imaginons que, tandis que je parle justement de Jules avec Nadia, nous voyions approcher M. Dupont. Il m'est tout à fait possible de m'exclamer :

(26) Regarde, il y a « Dulong » qui arrive.

Selon Recanati, l'énoncé produit dans ces circonstances est vrai. Mais cette vérité ne peut pas s'expliquer par un enrichissement pragmatique: il y a " Dulong " qui arrive (qui exprime la proposition selon laquelle Dupont arrive) n'implique pas sémantiquement il y a Dulong qui arrive. Recanati pose alors que la séquence "Dulong » reçoit son interprétation (ici : un référent) en fonction d'une autre langue (ici : l'idiolecte de Jules) que le reste de la phrase. Ceci a pour effet de modifier non seulement le contenu intuitif de l'énoncé mais aussi son contenu compositionnel! Néanmoins, cette modification n'est pas réellement sémantique mais bien plutôt «pré-sémantique ». En d'autres termes, c'est au cours de la phase où le destinataire établit quelle phrase a été énoncée (la phase de désambiguïsation, qui est de nature pragmatique) que le changement de langue opère. Donc, même si l'impact des guillemets se voit ici reflété dans les conditions de vérité sémantiques, (26) n'est pas un contre-exemple à l'explication pragmatique de Recanati.

Illustrons maintenant le «changement de situation ». L'exemple est en anglais, langue dans laquelle ce phénomène est plus fréquent :

(27) Wright won't disclose how much the Nike deal is worth, saying only that "they treat me well". (The Face, septembre $93: 55$ ) 
Cet exemple est non-cumulatif parce que (27) n'implique pas sémantiquement (27') :

(27') Wright won't disclose how much the Nike deal is worth, saying only that they treat me well.

En (27), me réfère à Wright. En (27'), me réfère au journaliste qui énonce la phrase entière. Ces exemples, parce qu'ils sont légion, même dans la presse la plus réputée des pays anglo-saxons, ne peuvent être rejetés sous prétexte d'agrammaticalité. Il faut donc leur trouver une explication. Celle-ci s'appuiera de nouveau sur le changement d'un des éléments du contexte, en l'occurrence la situation d'énonciation. C'est par rapport à une autre situation (celle où s'exprimait Wright - pas celle où le journaliste rédigeait son article) que le pronom me se voit assigner un référent. Encore une fois, ce changement dans le contexte a lieu pré-sémantiquement. Encore une fois, la modification observée $\mathrm{du}$ contenu compositionnel ne remet pas en question la thèse pragmatique de Recanati.

Passons enfin au «changement de circonstance d'évaluation». Nous reprenons ici un exemple discuté par Recanati lui-même (2001 : 677-79) :

Supposons que Jean et moi sachions (et sachions que l'autre sait) que Pierre croit à tort que Marie est ma sœur. Apercevant Marie, Jean me dit :

[(28)] Regarde ! « Ta sœur » vient vers nous.

En employant cette description incorrecte ( Ta sœur ») pour désigner Marie, Jean ironise sur la méprise de Pierre. Que nous avons ici affaire à un hybride non cumulatif est démontré par le fait que [(28)] n'implique pas sémantiquement

$\left[\left(28^{\prime}\right)\right]$ Ta sœur vient vers nous.

Comme on le voit, cet exemple ressemble à s'y méprendre à (26). Mais ne nous méprenons pas. Il n'est pas possible ici d'invoquer un changement de langue : l'expression «Ta sœur» n'a certainement pas une autre signification dans l'idiolecte de Pierre. Non, ce qui se passe ici, c'est que «Ta sœur» se voit attribuer une extension par rapport à un autre «monde» que le monde actuel (dans lequel Marie n'est pas la sœur du destinataire). Cet autre monde, c'est le «monde de croyance» de Pierre, puisque c'est bien dans ce monde-là que le destinataire a pour sœur Marie. Il ne s'agit pas non plus d'un changement dans la situation d'énonciation: il n'y en a qu'une en (28) : le déictique ta est bien interprété comme désignant le destinataire de l'énoncé (28), pas d'un quelconque énoncé antérieur.

Comme pour les autres glissements contextuels, ce changement dans la circonstance d'évaluation modifie le contenu compositionnel de (28) sans toutefois menacer la théorie de Recanati. Tout cela, à condition, bien entendu, 
que l'explication fondée sur les changements contextuels soit elle-même correcte. Ici, un important travail de réflexion et d'observation de données doit encore être accompli.

\section{Conclusions}

Le tableau que nous avons brossé se voulait aussi complet que possible, mais il a été difficile de ne pas laisser parler nos propres préférences. Nous espérons pourtant avoir réussi à éclairer tant les origines de la réflexion philosophique sur l'autonymie que les infléchissements qu'elle a connus. On a ainsi pu observer une transition graduelle d'un discours prescriptif motivé par des préoccupations logiciennes à une approche plus descriptive motivée par une attention croissante à l'étonnante variété des usages citationnels. Cette transformation s'est accompagnée d'une évolution de l'objet au centre des préoccupations. On est passé de l'autonyme de mot à l'autonyme de phrase (discours direct), puis à toutes les formes d'hybridité, dont on est loin d'avoir épuisé la richesse. Les configurations sur lesquelles se penchent actuellement les chercheurs sont d'un degré de complexité extrême en comparaison des autonymes. Pourtant, il nous semble que si l'on ne s'était pas d'abord attelé à donner une assise solide à la théorie de l'autonymie, on n'aurait jamais été en mesure de proposer des explications (pas seulement des descriptions) pour les configurations hybrides. Le fait que les branches les plus formelles de la linguistique et de la philosophie du langage parviennent maintenant à modéliser des hybrides complexes est un indice des progrès enregistrés dans le domaine (voir Potts 2004, Cumming 2005, Geurts \& Maier 2005).

Il reste toutefois beaucoup de travail à faire. En particulier, il s'agit de se pencher sur le rôle des données empiriques. Les quelques réflexions qui suivent vont toutes dans le sens d'un rapprochement plus étroit encore entre philosophes du langage et linguistes.

Malgré l'évolution du prescriptif au descriptif évoquée ci-dessus, on se doit de constater que les philosophes du langage usent encore souvent de corpus assez pauvres. Ceci n'est pas en soi une mauvaise chose. Entre autres, il nous semble que lorsqu'on oeuvre à construire une théorie, on a tout intérêt à se focaliser sur un nombre restreint d'exemples dont on a de bonnes raisons de penser qu'ils sont les plus pertinents. Par la suite, toutefois, il est recommandable de se « lancer dans le siècle » et d'y tester son édifice conceptuel. A cet égard, il ne serait pas inutile que les philosophes prêtent enfin attention aux données de l'oral. Jusqu'ici, ils sont restés très attachés à la langue écrite. Ceci a créé une situation où les marques (écrites) de la citation ont acquis une importance qui peut paraître démesurée.

Certains philosophes sont conscients du problème... et sont prêts, dès lors, à admettre que leur théorie est davantage une théorie des guillemets que de la citation dans son ensemble. Face à cette situation, un linguiste pourrait estimer 
que le philosophe prend plaisir à mettre la charrue avant les bœufs, voire que son entreprise est vouée à l'échec, étant donné la profusion d'exemples de citations sans marques. Au lieu de cela, il pourrait sembler souhaitable d'édifier une théorie générale de la citation, au sein de laquelle on rendrait ensuite compte du rôle particulier des guillemets.

De fait, pareilles théories existent déjà. Certains auteurs, comme Saka et Recanati ont développé des cadres parfaitement compatibles avec une approche généralisante. Ainsi, même s'ils parlent abondamment des guillemets, ils ne leur font jouer aucun rôle théorique essentiel : les guillemets sont réduits au statut d'indicateurs pragmatiques de phénomènes qui peuvent très bien se produire en leur absence. Ces auteurs-là peuvent à juste titre ne pas se sentir concernés par la critique.

Mais tous les chercheurs, même ceux qui n'ont pas tout misé sur les guillemets, auraient tout intérêt à prêter une oreille plus attentive à l'oral. Il est une question qui revient sans cesse sur le tapis mais qui jamais n'est réellement explorée : y a-t-il à l'oral des marques semblables aux guillemets, autrement dit, des marques qui ne sont pas simplement paralinguistiques, qui possèdent une signification linguistique conventionnelle? Le consensus est que pareilles marques n'existent pas (Geach 1957: 81 ; Rey-Debove 1978 : 73-74; Goldstein 1984:4; Washington 1992:588; C\&L 1997:431fn; Saka 1998:118; Recanati $2001: 661) \ldots$ mais personne n'est encore allé vérifier. Ce qui est quelque peu curieux puisque, s'il s'avérait que les guillemets ont un analogue oral, l'engouement des philosophes pour ces petites marques n'en deviendrait que plus justifiable. Il faut noter qu'un travail de ce type a été entrepris sur le discours direct par des linguistes de l'Université de Constance en Allemagne (Helga Kotthof, Elizabeth Couper-Kuhlen, Gabriele Klewitz) et les premiers résultats sont intrigants.

Enfin, il serait utile que les philosophes prêtent attention à une autre préoccupation typique des linguistes: la grammaticalité. A la période prescriptive, la question ne se posait pas. Mais l'attention croissante à l'usage linguistique réel l'a graduellement placée aux centre des préoccupations. Pourtant, on a un peu l'impression que pendant longtemps la question de la grammaticalité a gardé une fonction surtout rhétorique: on brandissait facilement l'argument de l'agrammaticalité (ou des doutes sur la grammaticalité) dès que l'on avait affaire à des données embarrassantes. Cette attitude semble vouée à disparaître, mais on peut légitimement s'étonner que les philosophes n'aient pas davantage réfléchi (ou demandé aux linguistes de réfléchir) à l'acceptabilité de certains des énoncés sur lesquels ils travaillent. Après tout, l'heure est à l'intégration; en particulier on voudrait que les analyses sémantiques puissent s'articuler aux analyses des syntacticiens. Nous terminerons sur un exemple. On trouve fréquemment des citations dans une langue différente de celle de la phrase citante. Parfois, ces citations sont des 
hybrides, à savoir qu'elles sont employées en même temps qu'elles sont mentionnées :

This is the France that has fueled the politics of "la fracture sociale" and of urban "insécurité". (Webpage)

Stendhal writes that she was "amoureuse de l'amour", being an enchanting and passionate actress. (Webpage)

Que veut dire " employer une séquence en français » dans un énoncé en anglais ? Quelle sorte de grammaire peut rendre compte de ces énoncés qui semblent néanmoins bien formés? Leur grammaticalité dépend-elle de la présence des guillemets (on y revient) ? Voilà quelques questions auxquelles philosophes et linguistes devront se confronter à l'avenir.

\author{
Institut Jean Nicod, CNRS-EHESS-ENS \\ phdebrab@yahoo.co.uk
}

\title{
Références :
}

Aввотт, Barbara (2005), « Some notes on quotation », Belgian Journal of Linguistics, $17,13-26$.

Atherton, Catherine (1993), The Stoics on Ambiguity, Cambridge/New York, Cambridge University Press, Cambridge Classical Studies.

BENBAJI, Yitzhak (2005), "Who needs semantics of quotation marks?», Belgian Journal of Linguistics, 17, 27-49.

BENNETT, Jonathan (1988), « Quotation », Noûs, 22, 399-418.

BYNUM, Terrell Ward (1972), « Biography and introduction », in Gottlob FREGE (1972), Conceptual Notation, and related articles, Oxford, Clarendon Press.

CAPPELEN, Herman \& Ernie LEPORE (1997), « Varieties of quotation », Mind, 106, 42950.

CAPPELEN, Herman \& Ernie LEPORE (1999), «Using, mentioning and quoting : a reply to Saka », Mind, 108, 741-50.

CAPPELEN, Herman \& Ernie LEPORE (2005), «Varieties of quotation revisited», Belgian Journal of Linguistics, 17, 51-75.

CARNAP, Rudolf (1934), Logische Syntax der Sprache, Vienne, Julius Springer, Schriften zur wissenschaftlichen Weltauffassung, 8.

CARnap, Rudolf (1937), The Logical Syntax of Language, trad. Amethe SMEATON, Londres, Kegan Paul.

CARnAP, Rudolf (1947), Meaning and Necessity. A Study in Semantics and Modal Logic, Chicago, The University of Chicago Press.

CARNAP, Rudolf (1948 (1942)), Introduction to Semantics, Cambridge, Mass., Harvard University Press. 
CARnAP, Rudolf (1950), Logical Foundations of Probability, Chicago, The University of Chicago Press.

CHURCH, Alonzo (1956), Introduction to Mathematical Logic, vol. 1, Princeton, Princeton University Press.

CHRISTENSEN, Niels Egmont (1967), «The alleged distinction between use and mention », Philosophical Review, 76, 358-67.

ClarK, Herbert H. \& Richard J. GERRIG (1990), «Quotations as demonstrations », Language, 66, 764-805.

CRAIG, Edward (sous la direction de) (1998), Routledge Encyclopedia of Philosophy, 10 vol., Londres/New York, Routledge.

CuMMING, Sam (2005), Belgian Journal of Linguistics, 17, 77-88.

DAVIDSON, Donald (1979), " Quotation », Theory and Decision, 11, 27-40.

DE BRABANTER, Philippe (à paraître), " Metalinguistic demonstrations and reference », in Maria José FrAPOLLI (éd.), Saying, Meaning and Referring. Essays on François Recanati's Philosophy of Language, Palgrave.

EDWARDS, Paul (sous la direction de) (1967), The Encyclopedia of Philosophy, New York, Macmillan \& The Free Press ; Londres, Collier-Macmillan.

GARCIA-CARPINTERO, Manuel (1994), «Ostensive signs : against the identity theory of quotation », The Journal of Philosophy, 91, 253-64.

GARCIA-CARPINTERO, Manuel (2005), «Double-duty quotation: the deferred ostension account », Belgian Journal of Linguistics, 17, 89-108.

GARVER, Newton (1965), "Varieties of use and mention», Philosophy and Phenomenological Research, 26, 230-38.

GEACH, Peter T. (1957), Mental Acts. Their Content and Their Objects, Londres, Routledge \& Kegan Paul.

GEURTS, Bart \& Emar MAIER (2005), "Quotation in context », Belgian Journal of Linguistics, 17, 109-128.

GómeZ-Torrente, Mario (2001), «Quotation revisited», Philosophical Studies, 102, $123-53$.

GÓMEZ-TORRENTE, Mario (2005), « Remarks on impure quotation », Belgian Journal of Linguistics, 17, 129-151.

IMBERT, Claude (1971), "Introduction», in Gottlob FREGE, Ecrits logiques et philosophiques, trad. Claude IMBERT, Paris, Seuil, coll. Points. Essais, 296.

LAMARQUE, Peter V. (ed.) (1997), Concise Encyclopedia of Philosophy of Language, Oxford/New York, Pergamon.

LEPORE, Ernie (1999), "The scope and limits of quotation », in Lewis Edwin HAHN (éd.), The Philosophy of Donald Davidson, Chicago/La Salle, Open Court, pp. 691714.

MAYENOWA, Maria Renata (1967), «Expressions guillemetées : contribution à l'étude de la sémantique du texte poétique », in To Honor Roman Jakobson. Essays on the occasion of his seventieth birthday, 11 october 1966, vol. 2, La Haye/Paris, Mouton, Janua linguarum. Series maior, 31-33, pp. 1315-27.

MitTElstrass, Jürgen (ed.) (1980-96), Enzyklopädie Philosophie und Wissenschaftstheorie, 4 vol., Mannheim, Bibliographisches Institut; Stuttgart/Weimar, Verlag J. B. Metzler. 
POTTS, Christopher (à paraître), "The dimensions of quotation », in Chris BARKER \& Pauline JACOBSON, (éds), Proceedings of the Workshop on Direct Compositionality. Cambridge, Mass., MIT Press.

PREDELLI, Stefano (2003), « Scare quotes and their relation to other semantic issues », Linguistics and Philosophy 26: 1-28.

QuINE, Willard Van Orman (1940), Mathematical Logic, Cambridge, Mass., Harvard University Press.

QuINE, Willard Van Orman (1953), From a Logical Point of View. 9 LogicoPhilosophical Essays, Cambridge, Mass., Harvard University Press.

QuINE, Willard Van Orman (1960), Word and Object, Londres, Wiley, Studies in Communication.

QuINE, Willard Van Orman (1974), Methods of Logic, 3è édition, Londres, Routledge \& Kegan Paul.

QuINE, Willard Van Orman (1976), The Ways of Paradox and Other Essays, 2è édition, Cambridge, Mass./Londres, Harvard University Press.

ReCANATI, François (1979), La transparence et l'énonciation. Pour introduire à la pragmatique, Paris, Seuil, coll. L'ordre philosophique.

RecanATI, François (2000), Oratio Obliqua, Oratio Recta: An Essay on Metarepresentation, Cambridge, Mass., MIT Press, Bradford Books.

ReCANATI, François (2001), « Open quotation », Mind, 110, 637-87.

REIMER, Marga (1996), "Quotation marks : demonstratives or demonstrations? », Analysis, 56, 131-41.

REIMER, Marga (2005), « Too counter-intuitive to believe? Pragmatic accounts of mixed quotation », Belgian Journal of Linguistics, 17, 167-186.

ReY-Debove, Josette (1978), Le Métalangage. Etude linguistique du discours sur le langage, Paris, Le Robert, coll. L'ordre des mots.

ReY-Debove, Josette (1997a), Le Métalangage. Etude linguistique du discours sur le langage, 2è édition augmentée, Paris, Armand Colin.

REY-Debove, Josette (1997b), "Les logiciens et le métalangage naturel », in REYDeBove, (1997a), pp. 293-304. Première parution : 1979, Histoire, épistémologie, langage, 1, 15-22.

RITTER, Joachim \& Karlfried GRÜNDER (éds.) (1971-2004), Historisches Wörterbuch der Philosophie, 12 vol., Bâle/Stuttgart, Schwabe \& Co AG Verlag.

ROSIER-CATACH, Irène (2004), « La suppositio materialis et la question de l'autonymie au Moyen-Âge », article accessible en ligne à www.cavi.univparis3.fr/ilpga/autonymie/theme1/rosiercatl.pdf

RUSSELL, Bertrand (1919). Introduction to Mathematical Philosophy, London: Allen \& Unwin.

SAKA, Paul (1998), « Quotation and the use-mention distinction », Mind, 107, 113-35.

SAKA, Paul (2005), «Quotational constructions », Belgian Journal of Linguistics, 17, $187-212$.

SEARLE, John R. (1969), Speech Acts: An Essay in the Philosophy of Language, Cambridge/New York, Cambridge University Press.

SEARle, John R. (1983), Intentionality. An Essay in the Philosophy of Mind, Cambridge, etc., Cambridge University Press.

SEYMOUR, Daniel (1996), « Content and quotation », Rivista di Linguistica, 8.2, 309-29. 
SIMCHEN, Ori (1999), "Quotational mixing of use and mention», Philosophical Quarterly, 49, 325-36.

SøRENSEN, Holger Steen (1958), Word-Classes in Modern English, with Special Reference to Proper Names, Copenhagen, G. E. C. Gad Publisher.

SøRENSEN, Holger Steen (1961), «An analysis of linguistic signs occurring in suppositio materialis or the meaning of quotation marks and their phonetic equivalents », Lingua, 10-2, 174-89.

STAINTON, Robert (1999), " Remarks on the syntax and semantics of mixed quotation », in Kimiko MURASUgi \& Robert STAINTON (éds), Philosophy and Linguistics, Boulder, Colorado, Westview Press, 259-78.

TARSKI, Alfred (1944), «The semantic conception of truth and the foundations of semantics », Journal of Philosophy and Phenomenological Research, 4, 341-75.

TARSKI, Alfred (1983), " The concept of truth in formalized languages ", in A. TARSKI, Logic, Semantics, Metamathematics. Papers from 1923 to 1938, trad. J. H. WOODGER, 2è édition avec une introduction de John CORCORAN, Indianapolis, Hackett Publishing Company, pp. 152-278.

Tsohatzidis, Savas L. (1998), « The hybrid theory of mixed quotation », Mind, 107, 661-64.

TsohatZIDIS, Savas L. (2005), "Lost hopes and mixed quotes », Belgian Journal of Linguistics, 17, 213-229.

WASHINGTON, Corey (1992), «Identity theory of quotation », Journal of Philosophy, 89, 582-605.

WASHINGTON, Corey (1998), "Use/mention distinction and quotation », in E. CRAIG (dir.), vol. 9, pp. 548-51. 